\title{
Computationally efficient analysis of extraordinary optical transmission through infinite and truncated subwavelength hole arrays
}

\author{
Miguel Camacho, ${ }^{*}$ Rafael R. Boix, ${ }^{\dagger}$ and Francisco Medina ${ }^{\ddagger}$ \\ Department of Electronics and Electromagnetics, College of Physics, Av. Reina Mercedes s/n, 41012 Seville, Spain
}

(Received 7 November 2015; revised manuscript received 30 April 2016; published 22 June 2016)

\begin{abstract}
The authors present a computationally efficient technique for the analysis of extraordinary transmission through both infinite and truncated periodic arrays of slots in perfect conductor screens of negligible thickness. An integral equation is obtained for the tangential electric field in the slots both in the infinite case and in the truncated case. The unknown functions are expressed as linear combinations of known basis functions, and the unknown weight coefficients are determined by means of Galerkin's method. The coefficients of Galerkin's matrix are obtained in the spatial domain in terms of double finite integrals containing the Green's functions (which, in the infinite case, is efficiently computed by means of Ewald's method) times cross-correlations between both the basis functions and their divergences. The computation in the spatial domain is an efficient alternative to the direct computation in the spectral domain since this latter approach involves the determination of either slowly convergent double infinite summations (infinite case) or slowly convergent double infinite integrals (truncated case). The results obtained are validated by means of commercial software, and it is found that the integral equation technique presented in this paper is at least two orders of magnitude faster than commercial software for a similar accuracy. It is also shown that the phenomena related to periodicity such as extraordinary transmission and Wood's anomaly start to appear in the truncated case for arrays with more than $100(10 \times 10)$ slots.
\end{abstract}

DOI: 10.1103/PhysRevE.93.063312

\section{INTRODUCTION}

The study of the transmission of electromagnetic waves through periodically perforated metal screens experienced an explosive growth in connection with the discovery of the so called extraordinary optical transmission at the turn of the 21 st century [1]. This phenomenon is well understood at present, and it is commonly explained in terms of the interaction of an impinging uniform plane wave with surface waves (spoof or designer plasmons $[2,3]$ ) supported by the periodically structured metal surface [4-7]. Although the first studies on extraordinary transmission focused their attention on optical frequencies, it soon became clear that this phenomenon also takes place at millimeter-wave or microwave frequencies [8,9]. This extension of the frequency range is not trivial since the behavior of metals at optical and microwave frequencies is quite different. Metals at microwave frequencies behave as quasiperfect conductors, while their behavior at the optical range is better described in terms of a lossy plasma. What happens in practice is that extraordinary transmission can be supported even by strictly perfect conductor screens, provided they are periodically perforated with subwavelength apertures [10]. It is the periodicity of the distribution of holes that is responsible for the existence of enhanced transmission peaks, rather than the specific constitutive relation of the metal at the operation frequency. The behavior of the metal has some influence, but it is not the main parameter to be taken into account. Moreover, although most of the papers published on the topic consider electrically thick metal slabs, extraordinary

\footnotetext{
*Also at Department of Physics and Astronomy, College of Engineering, Mathematics and Physical Sciences, University of Exeter, Exeter, Devon EX4, UK; mc586@exeter.ac.uk

†boix@us.es

${ }^{\ddagger}$ medina@us.es
}

transmission is present in two-dimensional periodic arrays of subwavelength apertures in metal screens of negligible thickness. The poor transmissivity through electrically small holes is only partially attributable to the fact that those holes are waveguides below cutoff. Poor transmissivity is also a feature of small apertures in zero-thickness metal screens due to the poor coupling provided by the small apertures to the radiated field [11]. Thus, the simplest possible structure exhibiting extraordinary transmission might be a two-dimensional periodic array of small apertures in a perfect conductor screen of negligible thickness. A thin holey metal screen operated at microwave frequencies could be reasonably approximated by such a model. This kind of structure is well known among microwave and antenna engineers, since they can be seen as the simplest version of a pass-band frequency-selective surface (FSS) [12-14].

The main difference between a FSS and an extraordinary transmission structure with similar geometry comes from the size of the apertures. In FSS applications, the apertures are electrically large enough to resonate at frequencies below the onset of the diffraction regime. The resonant frequency (for which total transmission is observed) is mainly controlled by the geometry of the apertures. In contrast, if the apertures are electrically small, a total extraordinary transmission peak can be observed at frequencies slightly below the first RayleighWood anomaly, which is controlled by the period of the structure rather than the size of the aperture. In any of those regimes, the characterization of the periodic system can be carried out by means of highly efficient numerical methods based on the solution of the electric field integral equation (EFIE) for the tangential components of the aperture electric field. Schuchinsky et al. provide in [15] an excellent example of the implementation of this method for a complex periodic layered structure involving several conducting surfaces with apertures. In this latter paper, the authors deal with infinite periodic structures since this makes it possible to reduce the 
analysis of the periodic structures to one single cell, which is an important simplification. A spectral domain approach combined with an analytical regularization of the EFIE is used in [15] for the efficient analysis of a large class of infinite periodic structures both in the resonant regime and in the extraordinary transmission regime. A different strategy is used in [16], where the authors use a hybrid spectral-spatial domain approach for the efficient solution of the EFIE arising in the analysis of multilayered infinite periodic structures with stacked metallic patches in the unit cell. In the current paper, we borrow some of the ideas presented in [16] to solve the EFIE involved in the analysis of an infinite periodic array of rectangular slots in a perfect electric conductor screen of negligible thickness. Since the direct application of the pure spectral domain approach leads to the determination of double infinite summations that are slowly convergent, in this paper a pure spatial domain approach is applied to the solution of the EFIE. Ewald's method is used in the computation of the free-space periodic Green's functions, in conjunction with tailored analytical-numerical techniques for the efficient determination of the four-dimensional (4D) singular integrals appearing in the application of Galerkin's method. Thanks to these two refinements in the solution of the EFIE, the spatial domain approach presented in this paper turns out to be more efficient than the classical pure spectral domain approach. Of course, more refined approaches in the spectral domain can be used to accelerate the computation of the slowly convergent double infinite summations, such as the application of Kummer's transformation [17] combined with either contour integration in the complex plane [18] or Poisson's formula [19] for the computation of the asymptotic summations.

Although most of the theoretical and numerical studies of extraordinary transmission structures have focused on structures with an infinite number of cells, this is just an approximation to real experiments in which the number of cells is finite. The investigation of truncation effects in periodic structures is relevant since it is important to know how the real finite structures are able to model the results provided by an infinite periodic model, e.g., the appearance of a clear extraordinary transmission peak. This is especially relevant for practical applications of this phenomenology in the implementation of physical devices. In fact, experimental and numerical simulations have been used to explore finite-size effects in two-dimensional arrays of apertures in metal slabs in the sub- $\mathrm{THz}$ region [20] and in the optical domain [21]. Also, a detailed semianalytical study of finite-size effects in one-dimensional arrays of slits in perfect conductor screens has been carried out in [22]. From the numerical point of view, the problem of the finite-size periodic array is much more challenging than that posed by the infinite array (restricted in practice to one single cell), especially when two-dimensional systems are considered. In this paper, the spatial domain EFIE method used in the analysis of an infinite periodic array of rectangular slots in a perfect electric conductor screen is readily adapted to handle the case in which the periodic array of rectangular slots is truncated. The numerical efficiency of the method makes it possible to deal with finite-size arrays containing from 10 to 10000 slots. While the CPU time involved in a frequency dependence study of the largest arrays may take a few minutes with our software, this may require many hours (even several days) when a commercial full-wave solver is employed. The application of the numerical method proposed in this paper has made it possible to understand the evolution of the frequency response of periodic twodimensional finite arrays of slots as the number of slots is systematically increased. This is something that may lead to a prohibitive computational effort if commercial software were to be used instead.

\section{INFINITE PERIODIC ARRAYS}

\section{A. Integral equation and Galerkin's method}

Figure 1 shows a perfectly conducting screen of negligible thickness located at the plane $z=0$ in free space. The screen contains an infinitely periodic array of rectangular slots. The unit cell of the periodic structure is a rectangle of dimensions $a \times b$, and the dimensions of the rectangular slot placed inside this unit cell are $w_{s} \times l_{s}\left(w_{s} \ll a\right)$.

In the following, a harmonic time dependence of the physical quantities of the type $e^{j \omega t}$ will be assumed and suppressed throughout. Let us consider a plane electromagnetic wave propagating along the positive $z$ direction in the half-space $z<0$, which impinges normally on the periodically perforated screen of Fig. 1. Let $\mathbf{E}_{i}=E_{0} e^{-j k_{0} z} \hat{\mathbf{x}}\left(k_{0}=\omega \sqrt{\mu_{0} \epsilon_{0}}\right)$ be the complex electric field of this wave polarized along the $x$ direction. In the absence of the slots, the incident wave will generate a surface electric current density on the screen $\mathbf{J}^{\text {as }}=\frac{2 E_{0}}{Z_{0}} \hat{\mathbf{x}}$, where $Z_{0}=\sqrt{\mu_{0} / \epsilon_{0}}$ is the free-space impedance. In the presence of the slots, the impinging wave induces a surface magnetic current-tangential component of the electric field-in the slots [23], and this magnetic current radiates a scattered field in both half-spaces $z<0$ and $z>0$. Let $\mathbf{E}_{t}^{\mathrm{sc}}(x, y, z=0)=E_{x}^{\mathrm{sc}}(x, y, z=0) \hat{\mathbf{x}}+E_{y}^{\mathrm{sc}}(x, y, z=0) \hat{\mathbf{y}}$ be the tangential scattering electric field existing in the slots, and let $\mathbf{M}^{\mathrm{sc}}(x, y)=\hat{\mathbf{z}} \times \mathbf{E}_{t}^{\mathrm{sc}}(x, y, z=0)$ be the magnetic current density in the slots. Since the surface electric current density

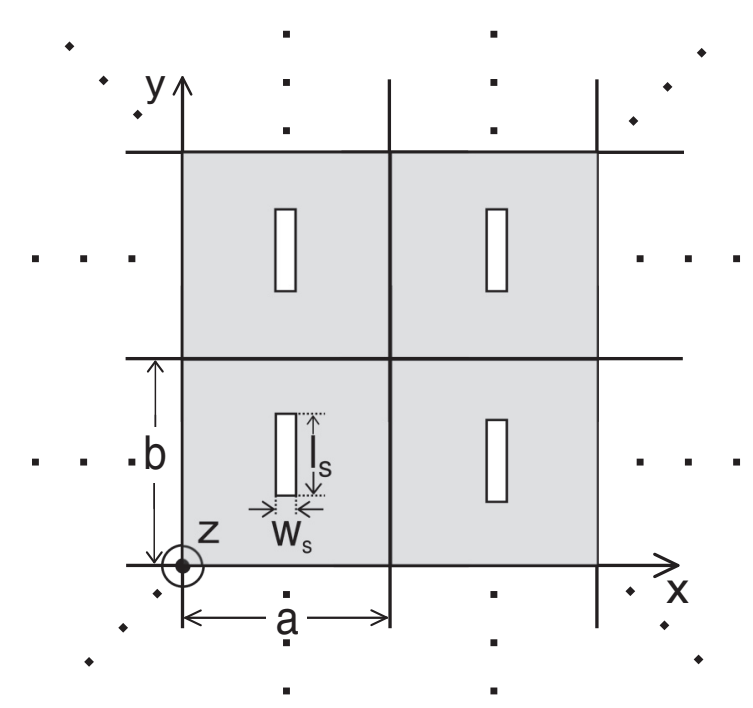

FIG. 1. Infinite periodic array of holes in a perfectly conducting screen of negligible thickness. The array is illuminated by a plane wave propagating along the $z$ direction (normal incidence). 
in the slots is zero, the following equation is satisfied:

$$
\begin{aligned}
& \mathbf{J}^{\mathrm{as}}+\int_{-\infty}^{\infty} \int_{-\infty}^{\infty} \overline{\mathbf{G}}_{M}\left(x-x^{\prime}, y-y^{\prime}\right) \\
& \cdot \mathbf{E}_{t}^{\mathrm{sc}}\left(x^{\prime}, y^{\prime}, z=0\right) d x^{\prime} d y^{\prime}=\mathbf{0} \quad(x, y) \in \text { slots },
\end{aligned}
$$

where $\overline{\mathbf{G}}_{M}(x, y)$ is the dyadic Green's function relating the surface electric current density on the conducting screen and the tangential electric field in the slots, and where the double integral has to be extended to all the slots in the infinite periodic structure (see Fig. 1). After using curl Maxwell's equations and boundary conditions on the conducting screen, the dyadic Green's function can be obtained in closed form, and it is given by

$$
\overline{\mathbf{G}}_{M}(x, y)=\left(\begin{array}{cc}
\left(k_{0}^{2}+\frac{\partial^{2}}{\partial y^{2}}\right) g(x, y) & -\frac{\partial^{2} g(x, y)}{\partial x \partial y} \\
-\frac{\partial^{2} g(x, y)}{\partial x \partial y} & \left(k_{0}^{2}+\frac{\partial^{2}}{\partial x^{2}}\right) g(x, y)
\end{array}\right),
$$

where

$$
g(x, y)=-\frac{j e^{-j k_{0} \sqrt{x^{2}+y^{2}}}}{\pi k_{0} Z_{0} \sqrt{x^{2}+y^{2}}} .
$$

Equation (1) is an integral equation for $\mathbf{E}_{t}^{\mathrm{sc}}(x, y, z=0)$. For normal plane-wave incidence, $\mathbf{E}_{t}^{\mathrm{sc}}(x, y, z=0)$ will be a periodic function of $x$ and $y$ [i.e., $\mathbf{E}_{t}^{\mathrm{sc}}(x+m a, y+n b, z=$ $\left.0)=\mathbf{E}_{t}^{\mathrm{sc}}(x, y, z=0) ; m, n=\cdots,-1,0,1, \ldots\right]$, and the integral equation can be reduced to

$$
\begin{aligned}
& \mathbf{J}^{\mathrm{as}}+\int_{0}^{a} \int_{0}^{b} \overline{\mathbf{G}}_{M}^{\mathrm{per}}\left(x-x^{\prime}, y-y^{\prime}\right) \\
& \cdot \mathbf{E}_{t}^{\mathrm{sc}}\left(x^{\prime}, y^{\prime}, z=0\right) d x^{\prime} d y^{\prime}=\mathbf{0}, \quad(x, y) \in \delta_{00},
\end{aligned}
$$

where $\delta_{00}$ is the slot $\left\{\left(a-w_{s}\right) / 2<x<\left(a+w_{s}\right) / 2\right.$; $\left.\left(b-l_{s}\right) / 2<y<\left(b+l_{s}\right) / 2\right\}$ of the periodic cell $C_{00}$ covering the rectangular domain $\{0 \leqslant x \leqslant a ; 0 \leqslant y \leqslant b\}$, and where $\overline{\mathbf{G}}_{M}^{\mathrm{per}}(x, y)$ is the periodic dyadic Green's function given by

$$
\overline{\mathbf{G}}_{M}^{\mathrm{per}}(x, y)=\sum_{m=-\infty}^{+\infty} \sum_{n=-\infty}^{+\infty} \overline{\mathbf{G}}_{M}(x-m a, y-n b) .
$$

To determine the value of $\mathbf{E}_{t}^{\mathrm{sc}}(x, y, z=0)$ in the slot $\delta_{00}$, we can apply the method of moments (MoM) [24] to the integral equation shown in (4). For that purpose, the tangential electric field in the slot is approximately expressed as a linear combination of known basis functions $\mathbf{b}_{j}(x, y)$ $\left(j=1, \ldots, N_{b}\right)$ as shown below,

$$
\mathbf{E}_{t}^{\mathrm{sc}}(x, y, z=0) \approx \sum_{j=1}^{N_{b}} e_{\infty, j} \mathbf{b}_{j}(x, y), \quad(x, y) \in \delta_{00},
$$

where $e_{\infty, j}$ are unknown constant coefficients. If (6) is substituted in (4), and Galerkin's version of MoM is used [i.e., the basis functions of (6) are used as weighting functions and (4) is projected over these weighting functions in the domain $C_{00}$, the following system of equations is obtained for the unknown coefficients $e_{\infty, j}$ :

$$
\sum_{j=1}^{N_{b}} \Gamma_{i j} e_{\infty, j}=C_{i} \quad\left(i=1, \ldots, N_{b}\right),
$$

where

$$
\begin{aligned}
\Gamma_{i j}= & \int_{0}^{a} \int_{0}^{b} \mathbf{b}_{i}^{*}(x, y) \cdot\left[\int_{0}^{a} \int_{0}^{b} \overline{\mathbf{G}}_{M}^{\mathrm{per}}\left(x-x^{\prime}, y-y^{\prime}\right)\right. \\
& \left.\cdot \mathbf{b}_{j}\left(x^{\prime}, y^{\prime}\right) d x^{\prime} d y^{\prime}\right] d x d y
\end{aligned}
$$

and where

$$
C_{i}=-\left(\int_{0}^{a} \int_{0}^{b} \mathbf{b}_{i}^{*}(x, y) d x d y\right) \cdot \mathbf{J}^{\mathrm{as}} .
$$

In principle, the calculation of Galerkin's matrix coefficients $\Gamma_{i j}$ of (8) is cumbersome since it involves the numerical determination of two nested double integrals, and the double infinite summation of (5) has to be computed every time the integrand is evaluated. Mittra et al. [13] suggest carrying out that computation in the spectral domain. Since $\mathbf{E}_{t}^{\mathrm{sc}}(x, y, z=0)$ is a periodic function of $x$ and $y$, the basis functions $\mathbf{b}_{j}(x, y)$ of (8) should also be periodic functions of $x$ and $y$, and they should admit a Fourier series representation of the type

$$
\mathbf{b}_{j}(x, y)=\sum_{m=-\infty}^{+\infty} \sum_{n=-\infty}^{+\infty} \widetilde{\mathbf{b}}_{j}\left(k_{x m}, k_{y n}\right) e^{j\left(k_{x m} x+k_{y n} y\right)},
$$

where $k_{x m}=\frac{2 \pi m}{a}$ and $k_{y n}=\frac{2 \pi n}{b}$, and where $\widetilde{\mathbf{b}}_{j}\left(k_{x m}, k_{y n}\right)$ is the 2D discrete Fourier transform of $\mathbf{b}_{j}(x, y)$, which is given by

$$
\widetilde{\mathbf{b}}_{j}\left(k_{x m}, k_{y n}\right)=\frac{1}{a b} \int_{0}^{a} \int_{0}^{b} \mathbf{b}_{j}(x, y) e^{-j\left(k_{x m} x+k_{y n} y\right)} d x d y .
$$

It can be easily shown that the term between square brackets in (8) is also a periodic function of $x$ and $y$. If both Parseval's theorem and the convolution theorem are applied to (8), after some manipulations it can be proven that the coefficients $\Gamma_{i j}$ can be rewritten in the spectral domain as

$$
\begin{aligned}
\Gamma_{i j}= & a b \sum_{m=-\infty}^{\infty} \sum_{n=-\infty}^{\infty} \widetilde{\mathbf{b}}_{i}^{*}\left(k_{x m}, k_{y n}\right) \\
& \cdot\left[\widetilde{\mathbf{G}}_{M}\left(k_{x}=k_{x m}, k_{y}=k_{y n}\right) \cdot \widetilde{\mathbf{b}}_{j}\left(k_{x m}, k_{y n}\right)\right],
\end{aligned}
$$

where $\widetilde{\mathbf{G}}_{M}\left(k_{x}, k_{y}\right)$ is the $2 \mathrm{D}$ continuous Fourier transform of the dyadic Green's function $\overline{\mathbf{G}}_{M}(x, y)$ of (2) and (3). According to Eq. (4.302) of [25], this Fourier transform can be obtained as

$$
\widetilde{\mathbf{G}}_{M}\left(k_{x}, k_{y}\right)=\frac{-2}{k_{0} Z_{0} k_{z}}\left(\begin{array}{cc}
k_{0}^{2}-k_{y}^{2} & k_{x} k_{y} \\
k_{x} k_{y} & k_{0}^{2}-k_{x}^{2}
\end{array}\right),
$$

where $k_{z}=\sqrt{k_{0}^{2}-k_{x}^{2}-k_{y}^{2}}$ when $k_{x}^{2}+k_{y}^{2}<k_{0}^{2}$ and $k_{z}=$ $-j \sqrt{k_{x}^{2}+k_{y}^{2}-k_{0}^{2}}$ when $k_{x}^{2}+k_{y}^{2}>k_{0}^{2}$ (this latter sign choice for the square root is very important since it makes it possible that the scattered field satisfies the radiation condition for $z \rightarrow \pm \infty$ ). According to (11), the constant terms $C_{i}$ of (9) can be rewritten in the spectral domain as

$$
C_{i}=-a b\left(\widetilde{\mathbf{b}}_{i}^{*}\left(k_{x m}=0, k_{y n}=0\right) \cdot \mathbf{J}^{\mathrm{as}}\right) .
$$

Although Eq. (12) seems to be much more convenient than (8) for the computation of $\Gamma_{i j}$, the double infinite summations of (12) usually converge very slowly, and a high number of terms has to be retained in order to obtain the resulting 
series with a reasonable accuracy. Some techniques have been applied to accelerate the convergence of these spectral domain series, such as Kummer's transformation [18,19]. There is an alternative efficient way of computing $\Gamma_{i j}$ in the spatial domain by means of Eq. (8). Let $\mathbf{b}_{j}(x, y)=b_{j x}(x, y) \hat{\mathbf{x}}+b_{j y}(x, y) \hat{\mathbf{y}}$ be the $j$ th basis function for $\mathbf{E}_{t}^{\mathrm{sc}}(x, y, z=0)$ in (6) and let $\mathbf{m}_{j}(x, y)=-b_{j y}(x, y) \hat{\mathbf{x}}+b_{j x}(x, y) \hat{\mathbf{y}}$ be the corresponding basis function for the magnetic current density in the slots $\mathbf{M}^{\mathrm{sc}}(x, y)$. After some mathematical manipulations, $\Gamma_{i j}$ can be rewritten as

$$
\Gamma_{i j}=-\frac{4 j}{k_{0} Z_{0}} \int_{-w_{s}}^{+w_{s}} \int_{-l_{s}}^{+l_{s}}\left[k_{0}^{2} f_{i j}(x, y)-f_{i j}^{d d}(x, y)\right] G_{\mathrm{sca}}^{\mathrm{per}}(x, y) d x d y .
$$

The integrals of (15) are finite double integrals that have to be numerically computed. The functions $f_{i j}(x, y)$ and $f_{i j}^{d d}(x, y)$ appearing in the integrands of (15) are cross-correlations between the vector functions $\mathbf{m}_{i}(x, y)$ and $\mathbf{m}_{j}(x, y)$, and between their divergences. These cross-correlations are given by

$$
\begin{gathered}
f_{i j}(x, y)=\int_{\left(a-w_{s}\right) / 2}^{\left(a+w_{s}\right) / 2} \int_{\left(b-l_{s}\right) / 2}^{\left(b+l_{s}\right) / 2} \mathbf{m}_{i}^{*}(x+u, y+v) \cdot \mathbf{m}_{j}(u, v) d u d v, \\
f_{i j}^{d d}(x, y)=\int_{\left(a-w_{s}\right) / 2}^{\left(a+w_{s}\right) / 2} \int_{\left(b-l_{s}\right) / 2}^{\left(b+l_{s}\right) / 2}\left[\nabla \cdot \mathbf{m}_{i}(x+u, y+v)\right]^{*}\left[\nabla \cdot \mathbf{m}_{j}(u, v)\right] d u d v .
\end{gathered}
$$

The function $G_{\mathrm{sca}}^{\mathrm{per}}(x, y)$ of $(15)$ is the free-space scalar two-dimensional periodic Green's function given by

$$
G_{\mathrm{sca}}^{\mathrm{per}}(x, y)=\sum_{m=-\infty}^{\infty} \sum_{n=-\infty}^{\infty} \frac{e^{-j k_{0} \sqrt{(x-m a)^{2}+(y-n b)^{2}}}}{4 \pi \sqrt{(x-m a)^{2}+(y-n b)^{2}}} .
$$

It turns out that $G_{\text {sca }}^{\text {per }}(x, y)$ can be efficiently computed by means of Ewald's method [26,27] in terms of the following expression:

$$
\begin{aligned}
& G_{\mathrm{sca}}^{\mathrm{per}}(x, y)=\frac{1}{2 j a b} \sum_{m=-\infty}^{\infty} \sum_{n=-\infty}^{\infty} \frac{e^{j\left(k_{x m} x+k_{y n} y\right)}}{\sqrt{k_{0}^{2}-k_{x m}^{2}-k_{y n}^{2}}} \operatorname{erfc}\left(\frac{j \sqrt{k_{0}^{2}-k_{x m}^{2}-k_{y n}^{2}}}{2 E}\right) \\
& +\frac{1}{8 \pi} \sum_{m=-\infty}^{\infty} \sum_{n=-\infty}^{\infty} \frac{1}{\sqrt{(x-m a)^{2}+(y-n b)^{2}}}\left[e^{j k_{0} \sqrt{(x-m a)^{2}+(y-n b)^{2}}} \operatorname{erfc}\left(\sqrt{(x-m a)^{2}+(y-n b)^{2}} E+\frac{j k_{0}}{2 E}\right)\right. \\
& \left.+e^{-j k_{0} \sqrt{(x-m a)^{2}+(y-n b)^{2}}} \operatorname{erfc}\left(\sqrt{(x-m a)^{2}+(y-n b)^{2}} E-\frac{j k_{0}}{2 E}\right)\right]
\end{aligned}
$$

where $\operatorname{erfc}(z)$ is the complementary error function of complex argument, and where the splitting parameter $E$ must be chosen as shown in [28]. As is well known, the two double infinite summations of (19) present Gaussian convergence, and it usually suffices to retain at most 50 terms in each double summation to obtain the series with machine-precision accuracy. If the basis functions $\mathbf{b}_{j}(x, y)$ of (6) are chosen in such a way that the cross-correlations of (16) and (17) can be obtained in closed form, the numerical computation of the integral in (15) provides a very convenient way to compute $\Gamma_{i j}$ since the integration domain of (15) is small [at least by comparison with the infinite summation domain of (12)], and the computation of $G_{\mathrm{sca}}^{\mathrm{per}}(x, y)$ can be efficiently carried out by means of (19). It should be pointed out that the integrands of (15) contain singularities that may have a deleterious effect on the numerical integration process. However, these singularities can be adequately handled, as will be shown in Sec. II C.

\section{B. Computation of transmission and reflection coefficients}

The fields scattered by the periodically perforated screen of Fig. 1 in the half-spaces $z<0$ and $z>0$ are periodic functions of $x$ and $y$, and they can be expressed in terms of Fourier series as shown below,

$$
\begin{aligned}
\mathbf{E}^{\mathrm{sc}}(x, y, z \lessgtr 0)= & \sum_{m=-\infty}^{+\infty} \sum_{n=-\infty}^{+\infty}\left[\widetilde{\mathbf{E}}_{t}^{\mathrm{sc}}\left(k_{x m}, k_{y n}, z=0\right)\right. \\
& \left.+\widetilde{E}_{z}^{\mathrm{sc}}\left(k_{x m}, k_{y n}, z=0^{\mp}\right) \hat{\mathbf{z}}\right] e^{j\left(k_{x m} x+k_{y n} y\right)} e^{j k_{z, m n} z},
\end{aligned}
$$

where $k_{z, m n}=\sqrt{k_{0}^{2}-k_{x m}^{2}-k_{y n}^{2}}$ when $k_{x m}^{2}+k_{y n}^{2}<k_{0}^{2}$ and $k_{z, m n}=-j \sqrt{k_{x m}^{2}-k_{y n}^{2}-k_{0}^{2}}$ when $k_{x m}^{2}+k_{y n}^{2}>k_{0}^{2}$, and where $\widetilde{\mathbf{E}}_{t}^{\mathrm{sc}}\left(k_{x m}, k_{y n}, z=0\right), \quad \widetilde{E}_{z}^{\mathrm{sc}}\left(k_{x m}, k_{y n}, z=0^{-}\right)$, and $\widetilde{E}_{z}^{\mathrm{sc}}\left(k_{x m}, k_{y n}, z=0^{+}\right)$are the discrete Fourier transforms of $\mathbf{E}_{t}^{\mathrm{sc}}(x, y, z=0), E_{z}^{\mathrm{sc}}\left(x, y, z=0^{-}\right)$, and $E_{z}^{\mathrm{sc}}\left(x, y, z=0^{+}\right)$. Equation (20) indicates that the electromagnetic fields scattered by the periodic screen of Fig. 1 are a linear combination of evanescent and propagating plane waves. The propagating waves are those for which the condition $k_{x m}^{2}+k_{y n}^{2}<k_{0}^{2}$ holds. Despite the value of the frequency, there will always be a scattered fundamental wave propagating in the negative $z$ direction for $z<0$ (reflected fundamental 
wave) and in the positive $z$ direction for $z>0$ (transmitted fundamental wave), which corresponds to the term $m=n=0$ in (20). Apart from this scattered fundamental wave, there will be scattered "grating lobes" with cutoff frequency $f_{m n}^{c}=c \sqrt{(m / a)^{2}+(n / b)^{2}}$, which propagate in the half-spaces $z<0$ (reflected grating lobe) and $z>0$ (transmitted grating lobe) along the directions given by the unit vectors,

$$
\hat{\mathbf{n}}_{\lessgtr, m n}=\frac{-k_{x m} \hat{\mathbf{x}}-k_{y n} \hat{\mathbf{y}} \mp k_{z, m n} \hat{\mathbf{z}}}{\sqrt{k_{x m}^{2}+k_{y n}^{2}+k_{z, m n}^{2}}} .
$$

Once the MoM described in Sec. II A has been applied and the system of Eqs. (7) has been solved to obtain the unknown coefficients $e_{\infty, j}$, in accordance with Eqs. (6) and (11), $\widetilde{\mathbf{E}}_{t}^{\mathrm{sc}}\left(k_{x m}, k_{y n}, z=0\right)$ will be given by

$$
\mathbf{E}_{t}^{\mathrm{sc}}\left(k_{x m}, k_{y n}, z=0\right)=\sum_{j=1}^{N_{b}} e_{\infty, j} \mathbf{b}_{j}\left(k_{x m}, k_{y n}\right),
$$

and using the divergence Maxwell's equation for the electric field in Eq. (20), it can be easily shown that $\widetilde{E}_{z}^{\mathrm{sc}}\left(k_{x m}, k_{y n}, z=\right.$ $\left.0^{-}\right)$and $\widetilde{E}_{z}^{\mathrm{sc}}\left(k_{x m}, k_{y n}, z=0^{+}\right)$can be expressed in terms of $e_{\infty, j}$ as shown below,

$$
\begin{aligned}
\widetilde{E}_{z}^{\mathrm{sc}}\left(k_{x m}, k_{y n}, z=0^{\mp}\right)= & \mp \frac{1}{k_{z, m n}}\left(k_{x m} \hat{\mathbf{x}}+k_{y n} \hat{\mathbf{y}}\right) \\
& \cdot\left(\sum_{j=1}^{N_{b}} e_{\infty, j} \mathbf{b}_{j}\left(k_{x m}, k_{y n}\right)\right) .
\end{aligned}
$$

A power reflection coefficient $R_{00}$ can be defined for the reflected fundamental wave as

$$
\begin{aligned}
R_{00} & =\frac{\left|\left[-E_{0} \hat{\mathbf{x}}+\widetilde{\mathbf{E}}_{t}^{\mathrm{sc}}\left(k_{x 0}, k_{y 0}, z=0\right)\right] e^{j k_{0} z}\right|^{2}}{\left|\mathbf{E}_{i}\right|^{2}} \\
& =\frac{\left|-E_{0} \hat{\mathbf{x}}+\sum_{j=1}^{N_{b}} e_{\infty, j} \mathbf{b}_{j}\left(k_{x 0}, k_{y 0}\right)\right|^{2}}{E_{0}^{2}},
\end{aligned}
$$

where the contribution of the reflected electric field in the absence of slots $\mathbf{E}_{r}^{\mathrm{as}}=-E_{0} e^{j k_{0} z} \hat{\mathbf{x}}$ has been included. Also, a power transmission coefficient $T_{00}$ can be defined for the transmitted fundamental wave as

$$
\begin{aligned}
T_{00} & =\frac{\left|\left[\widetilde{\mathbf{E}}_{t}^{\mathrm{sc}}\left(k_{x 0}, k_{y 0}, z=0\right)\right] e^{-j k_{0} z}\right|^{2}}{\left|\mathbf{E}_{i}\right|^{2}} \\
& =\frac{\left|\sum_{j=1}^{N_{b}} e_{\infty, j} \mathbf{b}_{j}\left(k_{x 0}, k_{y 0}\right)\right|^{2}}{E_{0}^{2}} .
\end{aligned}
$$

In the same way, a power reflection coefficient $R_{m n}$ can be defined for the power reflected by the grating lobe $m-n$ along the negative $z$ direction when frequency is above $f_{m n}^{c}$, and a power transmission coefficient $T_{m n}$ can be defined for the power transmitted by that grating lobe along the positive $z$ direction. $R_{m n}$ and $T_{m n}$ are given by the expressions

$$
\begin{aligned}
R_{m n}= & \frac{k_{z, m n}}{\sqrt{k_{x m}^{2}+k_{y n}^{2}+k_{z, m n}^{2}}} \\
& \times \frac{\left|\widetilde{\mathbf{E}}_{t}^{\mathrm{sc}}\left(k_{x m}, k_{y n}, z=0\right)\right|^{2}+\left|\widetilde{E}_{z}^{\mathrm{sc}}\left(k_{x m}, k_{y n}, z=0^{-}\right)\right|^{2}}{E_{0}^{2}}, \\
T_{m n}= & \frac{k_{z, m n}}{\sqrt{k_{x m}^{2}+k_{y n}^{2}+k_{z, m n}^{2}}} \\
& \times \frac{\left|\widetilde{\mathbf{E}}_{t}^{\mathrm{sc}}\left(k_{x m}, k_{y n}, z=0\right)\right|^{2}+\left|\widetilde{E}_{z}^{\mathrm{sc}}\left(k_{x m}, k_{y n}, z=0^{+}\right)\right|^{2}}{E_{0}^{2}}, \\
\tilde{x}^{\mathrm{sc}} & |m|+|n| \neq 0,
\end{aligned}
$$

where $\widetilde{\mathbf{E}}_{t}^{\mathrm{sc}}\left(k_{x m}, k_{y n}, z=0\right), \quad \widetilde{E}_{z}^{\mathrm{sc}}\left(k_{x m}, k_{y n}, z=0^{-}\right), \quad$ and $\widetilde{E}_{z}^{\mathrm{sc}}\left(k_{x m}, k_{y n}, z=0^{+}\right)$must be obtained by means of Eqs. (22) and (23).

Power conservation enforces the fulfillment of the following condition:

$$
\left.\sum_{m} \sum_{n}\left(R_{m n}+T_{m n}\right)\right|_{k_{x m}^{2}+k_{y n}^{2}<k_{0}^{2}}=1 .
$$

\section{Basis functions and computation of Galerkin's matrix coefficients}

The basis functions chosen for $\mathbf{E}_{t}^{\mathrm{sc}}(x, y, z=0)$ in the slot $\delta_{00}$ are

$$
\begin{aligned}
\mathbf{b}_{j}(x, y)= & g_{j x}^{e e}\left(x-\frac{a}{2}, y-\frac{b}{2}\right) \hat{\mathbf{x}}=q_{x}^{e}\left(x-\frac{a}{2}\right) r_{j x}^{e}\left(y-\frac{b}{2}\right) \hat{\mathbf{x}}=\frac{T_{0}\left(\frac{2}{w_{s}}\left(x-\frac{a}{2}\right)\right)}{\sqrt{1-\left[\frac{2}{w_{s}}\left(x-\frac{a}{2}\right)\right]^{2}}} \\
\times & U_{2(j-1)}\left[\frac{2}{l_{s}}\left(y-\frac{b}{2}\right)\right] \sqrt{1-\left[\frac{2}{l_{s}}\left(y-\frac{b}{2}\right)\right]^{2}} \hat{\mathbf{x}} \quad\left(j=1, \ldots, N_{b x}^{e e}\right), \\
\mathbf{b}_{N_{b x}^{e e}+j}(x, y)= & g_{j y}^{o o}\left(x-\frac{a}{2}, y-\frac{b}{2}\right) \hat{\mathbf{y}}=q_{y}^{o}\left(x-\frac{a}{2}\right) r_{j y}^{o}\left(y-\frac{b}{2}\right) \hat{\mathbf{y}}=U_{1}\left[\frac{2}{w_{s}}\left(x-\frac{a}{2}\right)\right] \\
& \times \sqrt{1-\left[\frac{2}{w_{s}}\left(x-\frac{a}{2}\right)\right]^{2}} \frac{T_{2 j-1}\left(\frac{2}{l_{s}}\left(y-\frac{b}{2}\right)\right)}{\sqrt{1-\left[\frac{2}{l_{s}}\left(y-\frac{b}{2}\right)\right]^{2}}} \hat{\mathbf{y}} \quad\left(j=1, \ldots, N_{b y}^{o o}\right),
\end{aligned}
$$

where $N_{b x}^{e e}+N_{b y}^{o o}=N_{b}$, and where $T_{0}(\cdot)\left[T_{2 j-1}(\cdot)\right]$ and $U_{2(j-1)}(\cdot)\left[U_{1}(\cdot)\right]$ are Chebyshev polynomials of the first and second kind, respectively. 
Lerer and Schuchinsky [29] claim that the basis functions of (29) and (30) provide an excellent approximation of the electric current density on planar rectangular metallizations. Therefore, by virtue of Babinet's principle [23], the same basis functions should provide a good approximation of the magnetic current density in the slots of the periodically perforated screen of Fig. 1. The reason why the weighted Chebyshev polynomials of (29) and (30) are very adequate basis functions for $\mathbf{E}_{t}^{\mathrm{sc}}(x, y, z=0)$ is that the kernel of the integral equation of (1) is dominated by logarithmic singularities in the neighborhood of the slot edges, and the weighted Chebyshev polynomials represent the complete orthonormal set of eigenfunctions for an integral equation with a logarithmic kernel. This causes the weighted Chebyshev polynomials to be the most pertinent basis functions for the electromagnetic analysis of structures containing perfect conductors of negligible thickness with edges as detailed in [29] and references therein. Note that the basis functions of (29) are all even functions of $x-a / 2$ and $y-b / 2$, and that the basis functions of (30) are all odd functions of $x-a / 2$ and $y-b / 2$. These constraints are imposed by the fact that the planes $x=m a+a / 2(m=\ldots,-1,0,1, \ldots)$ of Fig. 1 are all electric walls, and the planes $y=n b+b / 2$ $(n=\ldots,-1,0,1, \ldots)$ are all magnetic walls [10] under normal incidence conditions for the type of excitation we have assumed (electric field polarized along the $x$ direction).

Bearing in mind that $\frac{d}{d x}\left[U_{n-1}(x) \sqrt{1-x^{2}}\right]=-n \frac{T_{n}(x)}{\sqrt{1-x^{2}}}$ $(n \geqslant 1)$, the discrete Fourier transforms of (29) and (30) can be shown to be proportional to $J_{0}\left(\frac{k_{x m} w_{s}}{2}\right) J_{2 j-1}\left(\frac{k_{y n} l_{s}}{2}\right) / k_{y n}$ and $J_{2}\left(\frac{k_{x m} w_{s}}{2}\right) J_{2 j-1}\left(\frac{k_{y n} l_{s}}{2}\right) / k_{x m}$, respectively, where $J_{k}(\cdot)$ are Bessel functions of the first kind and order $k$ [see Eq. (7.355) of [30]]. When these Fourier transforms are introduced in (12), the terms of the series $\Gamma_{i j}$ will decay as $|m|^{-2}|n|^{-2}$ when $|m| \rightarrow \infty$ and $|n| \rightarrow \infty$, which indicates that expressions (12) for $\Gamma_{i j}$ are slowly convergent series, as mentioned above.

The best way to obtain the cross-correlations of (16) and (17) for the basis functions of (29) and (30) is to express these cross-correlations in terms of the inverse Fourier transform of their Fourier transform. After some mathematical manipulations, it can be shown that all these cross-correlations can be expressed as linear combinations of the functions of two variables,

$$
F_{p r, q v}(x, y)=t_{p r}\left(x, w_{s}\right) t_{q v}\left(y, l_{s}\right),
$$

where the functions $t_{p r}\left(x, w_{s}\right)$ and $t_{q v}\left(y, l_{s}\right)$ were defined in Eq. (45) of [31], and they can be expressed in terms of the following set of integrals:

$$
t_{k l}(z, w)=\frac{\pi}{4 w} j^{k-l} \int_{-\infty}^{+\infty} J_{k-1}(u) J_{l-1}(u) e^{-j \frac{2 z u}{w}} d u .
$$

The integrals of (32) can be obtained in closed form by means of the recurrent relations (46) to (53) of [31]. In particular, these integrals can all be written as linear combinations of complete elliptic integrals of the first and second kind [see Eqs. (8.111) and (8.112) of [30]].

When the cross-correlations $f_{i j}(x, y)$ and $f_{i j}^{d d}(x, y)$ for the basis functions (29) and (30) are introduced in (15), the coefficients $\Gamma_{i j}$ can all be written as linear combinations of the integrals

$$
\begin{aligned}
\Omega_{p r, q v} & =\int_{-w_{s}}^{+w_{s}} \int_{-l_{s}}^{+l_{s}} t_{p r}\left(x, w_{s}\right) t_{q v}\left(y, l_{s}\right) G_{\mathrm{sca}}^{\mathrm{per}}(x, y) d x d y \\
& =\int_{-w_{s}}^{+w_{s}} \int_{-l_{s}}^{+l_{s}} w_{p r, q v}(x, y) d x d y .
\end{aligned}
$$

The double integrals of (33) turn out to be zero when $p+r$ and/or $q+v$ are odd since in this case $w_{p r, q v}(x, y)$ is an odd function of $x$ and/or $y$, and the integration interval of (33) is symmetric around 0 with respect to both $x$ and $y$. When $p+r$ and $q+v$ are both even [which is the case encountered for the specific basis functions of (29) and (30)], the integrals of (33) have to be numerically computed. The problem is that the integrands shown in (33) have singularities that tend to limit the accuracy of numerical integration formulas. On the one hand, the scalar periodic Green's function $G_{\mathrm{sca}}^{\mathrm{per}}(x, y)$ has a singularity when $x=y=0$ [present in the term $m=n=0$ of (18)]. On the other hand, the computation of the functions $t_{p r}\left(x, w_{s}\right)$ and $t_{q v}\left(y, l_{s}\right)$ of (31) involves the determination of the complete elliptic integrals of the first kind $K\left(\sqrt{1-\left(x / w_{s}\right)^{2}}\right)$ and $K\left(\sqrt{1-\left(y / l_{s}\right)^{2}}\right)$, which have logarithmic singularities when $x=0$ and $y=0$, respectively [see Eq. (8.113.3) of [30]]. This means that all three functions $G_{\mathrm{sca}}^{\mathrm{per}}(x, y), t_{p r}\left(x, w_{s}\right)$, and $t_{q v}\left(y, l_{s}\right)$ contribute to the singularity at $x=y=0$, that $t_{p r}\left(x, w_{s}\right)$ introduces a logarithmic singularity at $(x=0, y)$ when $y \neq 0$, and that $t_{q v}\left(y, l_{s}\right)$ introduces a logarithmic singularity at $(x, y=0)$ when $x \neq 0$. The singularity at $x=$ $y=0$ can be removed by means of the singularity extraction technique. For that purpose, we define a function $w_{p r, q v}^{\text {sing }}(x, y)$ that reproduces the behavior of $w_{p r, q v}(x, y)$ around $x=y=0$ when $p+r$ and $q+v$ are both even as

$$
\begin{aligned}
\left.w_{p r, q v}(x, y)\right|_{x^{2}+y^{2} \rightarrow 0} \approx & w_{p r, q v}^{\mathrm{sing}}(x, y) \\
= & A_{p r, q v}^{1}+A_{p r, q v}^{2} \ln |x|+A_{p r, q v}^{3} \ln |y| \\
& +A_{p r, q v}^{4} \ln |x| \ln |y| \\
& +\left(A_{p r, q v}^{5}+A_{p r, q v}^{6} \ln |x|\right. \\
& \left.+A_{p r, q v}^{7} \ln |y|+A_{p r, q v}^{8} \ln |x| \ln |y|\right) \\
& \times \frac{1}{\sqrt{x^{2}+y^{2}}},
\end{aligned}
$$

where the constant coefficients $A_{p r, q v}^{i}(i=1, \ldots, 8)$ are obtained as shown in Appendix. Once the functions $w_{p r, q v}^{\mathrm{sing}}(x, y)$ are defined, we split the integrals of (33) for both $p+r$ and $q+v$ even in two parts as shown below,

$$
\begin{aligned}
\Omega_{p r, q v}= & \int_{-w_{s}}^{+w_{s}} \int_{-l_{s}}^{+l_{s}}\left[w_{p r, q v}(x, y)-w_{p r, q v}^{\mathrm{sing}}(x, y)\right] d x d y \\
& +\int_{-w_{s}}^{+w_{s}} \int_{-l_{s}}^{+l_{s}} w_{p r, q v}^{\mathrm{sing}}(x, y) d x d y .
\end{aligned}
$$

The integrand of the first integral of (35) does not contain any singularity at $x=y=0$ since it has been removed. However, this integrand still keeps the logarithmic singularities of the complete elliptic integrals at both $(x=0, y)$ when $y \neq 0$ and $(x, y=0)$ when $x \neq 0$. These integrals can be numerically computed in a very efficient way by using iterated 
Ma-Rokhlin-Wandzura (MRW) quadrature rules [32] since these quadrature rules are especially tailored to deal with logarithmic singularities. The integrand of the second integral of (35) includes all the singularities of $w_{p r, q v}(x, y)$ at $x=$ $y=0$. Fortunately, this second integral can be determined in a very efficient way as explained in Appendix. To sum up, Eq. (35) provides an expression for the numerical computation of $\Omega_{p r, q v}$, which avoids the integrand singularities problems of (33). This approach provides an efficient way of computing the coefficients $\Gamma_{i j}$ for the basis functions of (29) and (30) without resorting to the slowly convergent series of (12).

\section{TRUNCATED PERIODIC ARRAYS}

\section{A. Integral equation and Galerkin's method}

Figure 2 shows a truncated version of Fig. 1 in which a finite periodic array of $N \times N=M$ slots is placed in an infinite perfectly conducting plane of negligible thickness located at $z=0$. Let $\Omega_{j}$ be the periodic cell-rectangular domain of dimensions $a \times b$ [in which the $j$ th slot is located $(j=1, \ldots, M)]$, let $\eta_{j}$ be the rectangular domain of dimensions $w_{s} \times l_{s}\left(w_{s} \ll a\right)$ occupied by the $j$ th slot, and let $\left(x_{c j}, y_{c j}, z_{c j}=0\right)$ be the Cartesian coordinates of the geometrical center of $\eta_{j}$. Again, we are going to consider a plane wave propagating along the positive $z$ direction in the half-space $z<0$ that impinges normally on the periodically perforated screen of Fig. 2. Let $\mathbf{E}_{i}=E_{0} e^{-j k_{0} z} \hat{\mathbf{x}}$ be the electric field of the incident wave, and let $\mathbf{J}^{\text {as }}=\frac{2 E_{0}}{Z_{0}} \hat{\mathbf{x}}$ be the electric current density that would be produced on the conducting screen in the absence of the slots. By analogy with (1), the tangential scattering electric field excited on the $M$ slots by the incident wave, $\mathbf{E}_{t}^{\mathrm{sc}}(x, y, z=0)$, will be the solution of the

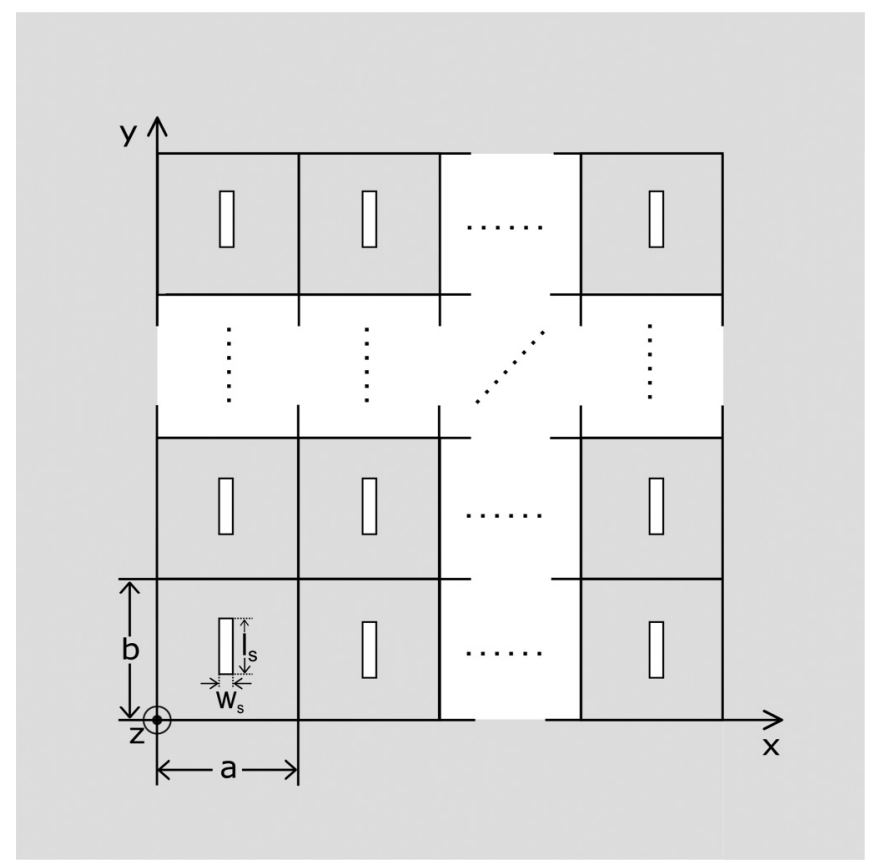

FIG. 2. Truncated periodic array of $N \times N$ holes in a perfectly conducting screen of negligible thickness. The array is illuminated by a plane wave propagating along the $z$ direction (normal incidence). following set of $M$ coupled integral equations:

$$
\begin{array}{r}
\mathbf{J}^{\mathrm{as}}+\sum_{j=1}^{M} \iint_{\eta_{j}} \overline{\mathbf{G}}_{M}\left(x-x^{\prime}, y-y^{\prime}\right) \\
\cdot \mathbf{E}_{t}^{\mathrm{sc}}\left(x^{\prime}, y^{\prime}, z=0\right) d x^{\prime} d y^{\prime}=\mathbf{0}, \quad(x, y) \in \eta_{i} \\
(i=1, \ldots, M),
\end{array}
$$

where $\overline{\mathbf{G}}_{M}(x, y)$ is the dyadic Green's function defined in (2) and (3). Since in the case of Fig. $2 \mathbf{E}_{t}^{\mathrm{sc}}(x, y, z=0)$ is no longer a periodic function of $x$ and $y$, the set of integral equations (36) cannot be reduced to one single periodic cell as we did in Sec. II with Eq. (4).

To determine the value of $\mathbf{E}_{t}^{\mathrm{sc}}(x, y, z=0)$ in $\eta_{j}(j=$ $1, \ldots, M)$, we will apply the MoM to each of the $M$ integral equations of (36). For that purpose, the tangential electric field in the $j$ th slot will be approximately expressed in terms of known basis functions $\mathbf{d}_{j l}(x, y)=d_{j l, x}(x, y) \hat{\mathbf{x}}+d_{j l, y}(x, y) \hat{\mathbf{y}}$ $\left(l=1, \ldots, N_{b}\right)$ as shown below,

$$
\mathbf{E}_{t}^{\mathrm{sc}}(x, y, z=0) \approx \sum_{l=1}^{N_{b}} e_{j l} \mathbf{d}_{j l}(x, y), \quad(x, y) \in \eta_{j},
$$

where $\mathbf{c}_{j l}(x, y)=-d_{j l, y}(x, y) \hat{\mathbf{x}}+d_{j l, x}(x, y) \hat{\mathbf{y}}$ is the $l$ th basis function for the magnetic current density, $\mathbf{M}^{\mathrm{sc}}(x, y)=\hat{\mathbf{z}} \times$ $\mathbf{E}_{t}^{\mathrm{sc}}(x, y, z=0)$, in the $j$ th slot.

Then, Eq. (37) will be substituted in (36), and Galerkin's version of MoM will be used to obtain a linear system of equations for the unknown coefficients $e_{j l}$, which is given by

$$
\sum_{j=1}^{M} \sum_{l=1}^{N_{b}} \Delta_{i j}^{k l} e_{j l}=p_{i k} \quad\left(i=1, \ldots, M ; k=1, \ldots, N_{b}\right),
$$

where

$$
\begin{aligned}
& \Delta_{i j}^{k l}= \iint_{\eta_{i}} \mathbf{d}_{i k}^{*}(x, y) \cdot\left[\iint_{\eta_{j}} \overline{\mathbf{G}}_{M}\left(x-x^{\prime}, y-y^{\prime}\right)\right. \\
&\left.\cdot \mathbf{d}_{j l}\left(x^{\prime}, y^{\prime}\right) d x^{\prime} d y^{\prime}\right] d x d y \\
& \quad\left(i, j=1, \ldots, M ; k, l=1, \ldots, N_{b}\right),
\end{aligned}
$$

and where

$$
\begin{aligned}
p_{i k}=-\left(\iint_{\eta_{i}} \mathbf{d}_{i k}^{*}(x, y) d x d y\right) \cdot \mathbf{J}^{\mathrm{as}} \\
\\
\quad\left(i=1, \ldots, M ; k=1, \ldots, N_{b}\right) .
\end{aligned}
$$

As happens with the infinite periodic case treated in Sec. IIC, Galerkin's matrix coefficients $\Delta_{i j}^{k l}$ of (8) can be computed in the spectral domain as

$$
\begin{aligned}
\Delta_{i j}^{k l}= & \frac{1}{4 \pi^{2}} \int_{-\infty}^{+\infty} \int_{-\infty}^{+\infty} \widetilde{\mathbf{d}}_{i k}^{*}\left(k_{x}, k_{y}\right) \\
& \cdot\left[\widetilde{\mathbf{G}}_{M}\left(k_{x}, k_{y}\right) \cdot \widetilde{\mathbf{d}}_{j l}\left(k_{x}, k_{y}\right)\right] d k_{x} d k_{y},
\end{aligned}
$$

where $\widetilde{\mathbf{G}}_{M}\left(k_{x}, k_{y}\right)$ is the spectral dyadic Green's function defined in (13) and $\widetilde{\mathbf{d}}^{i k}\left(k_{x}, k_{y}\right)$ is the $2 \mathrm{D}$ continuous Fourier 
transform of $\mathbf{d}_{i k}(x, y)$ given by

$$
\begin{gathered}
\tilde{\mathbf{d}}_{i k}\left(k_{x}, k_{y}\right)=\int_{-\infty}^{+\infty} \int_{-\infty}^{+\infty} \mathbf{d}_{i k}(x, y) e^{-j\left(k_{x} x+k_{y} y\right)} d x d y \\
\left(i=1, \ldots, M ; k=1, \ldots, N_{b}\right) .
\end{gathered}
$$

Equation (41) is not an efficient expression for the computation of $\Delta_{i j}^{k l}$ since it implies the numerical evaluation of a double integral with infinite limits (even though a change to polar spectral variables $k_{x}=k_{\rho} \cos k_{\varphi}$ and $k_{x}=k_{\rho} \sin k_{\varphi}$ is carried out, an integral with infinite limits is still left) and the integrands slowly decay as $k_{\rho}=\sqrt{k_{x}^{2}+k_{y}^{2}} \rightarrow \infty$. As in the case of Eq. (15) obtained in Sec. II, there is an alternative expression for the computation of $\Delta_{i j}^{k l}$ in the spatial domain, which is given by

$$
\begin{aligned}
\Delta_{i j}^{k l}= & -\frac{4 j}{k_{0} Z_{0}} \int_{\left(x_{c i}-x_{c j}\right)-w_{s}}^{\left(x_{c i}-x_{c j}\right)+w_{s}} \int_{\left(y_{c i}-y_{c j}\right)-l_{s}}^{\left(y_{c i}-y_{c j}\right)+l_{s}} \\
& {\left[k_{0}^{2} h_{i j}^{k l}(x, y)-h_{i j}^{k l, d d}(x, y)\right] G_{\mathrm{sca}}(x, y) d x d y, }
\end{aligned}
$$

where the integrals of (43) are finite double integrals that have to be numerically computed. By analogy with Eqs. (16) and (17), the functions $h_{i j}^{k l}(x, y)$ and $h_{i j}^{k l, d d}(x, y)$ of (43) are cross-correlations between the vector functions $\mathbf{c}_{i k}(x, y)$ and $\mathbf{c}_{j l}(x, y)$, and between their divergences. These cross-correlations are given by

$$
\begin{aligned}
h_{i j}^{k l}(x, y)= & \int_{x_{c j}-w_{s} / 2}^{x_{c j}+w_{s} / 2} \int_{y_{c j}-l_{s} / 2}^{y_{c j}+l_{s} / 2} \\
& \mathbf{c}_{i k}^{*}(x+u, y+v) \cdot \mathbf{c}_{j l}(u, v) d u d v,
\end{aligned}
$$

$$
\begin{aligned}
h_{i j}^{k l, d d}(x, y)= & \int_{x_{c j}-w_{s} / 2}^{x_{c j}+w_{s} / 2} \int_{y_{c j}-l_{s} / 2}^{y_{c j}+l_{s} / 2} \\
& {\left[\nabla \cdot \mathbf{c}_{i k}(x+u, y+v)\right]^{*}\left[\nabla \cdot \mathbf{c}_{j l}(u, v)\right] d u d v . }
\end{aligned}
$$

The function $G_{\text {sca }}(x, y)$ of (43) is the free-space nonperiodic scalar Green's function given by

$$
G_{\mathrm{sca}}(x, y)=\frac{e^{-j k_{0} \sqrt{x^{2}+y^{2}}}}{4 \pi \sqrt{x^{2}+y^{2}}} .
$$

\section{B. Computation of far field and effective receiving area}

Let $\quad \widetilde{\mathbf{E}}_{t}^{\mathrm{sc}}\left(k_{x}, k_{y}, z=0\right)=\widetilde{E}_{x}^{\mathrm{sc}}\left(k_{x}, k_{y}, z=0\right) \hat{\mathbf{x}}+\widetilde{E}_{y}^{\mathrm{sc}}\left(k_{x}, k_{y}, z=\right.$ $0) \hat{\mathbf{y}}$ be the $2 \mathrm{D}$ continuous Fourier transform of $\mathbf{E}_{t}^{\mathrm{sc}}(x, y, z=0)$. Since the conductor screen of Fig. 2 has been assumed to be a perfect electric conductor, $\mathbf{E}_{t}^{\mathrm{sc}}(x, y, z=0)$ will only be different from zero in the slots $\eta_{j}(j=1, \ldots, M)$ of the plane $z=0$. In accordance with this statement and in accordance with (37), we can write

$$
\widetilde{\mathbf{E}}_{t}^{\mathrm{sc}}\left(k_{x}, k_{y}, z=0\right)=\sum_{j=1}^{M} \sum_{l=1}^{N_{b}} e_{j l} \widetilde{\mathbf{d}}_{j l}\left(k_{x}, k_{y}\right),
$$

where the spectral functions $\widetilde{\mathbf{d}}_{j l}\left(k_{x}, k_{y}\right)$ have been defined in (42). If we follow the guidelines of Sec. 4.1 of [33], the scattered electric field radiated by the truncated periodic screen of Fig. 2 in the half-space $z>0$ can be obtained in spherical coordinates in terms of the components of $\widetilde{\mathbf{E}}^{\mathrm{sc}}\left(k_{x}, k_{y}, z=0\right)$ as shown below,

$$
\begin{aligned}
\left.\mathbf{E}^{\mathrm{sc}}(r \gg, \theta, \phi)\right|_{z>0}= & \frac{j k_{0} e^{-j k_{0} r}}{2 \pi r}\left[\left(\widetilde{E}_{x}^{\mathrm{sc}}\left(k_{x}=k_{0} \sin \theta \cos \phi, k_{y}=k_{0} \sin \theta \sin \phi, z=0\right) \cos \varphi\right.\right. \\
& \left.+\widetilde{E}_{y}^{\mathrm{sc}}\left(k_{x}=k_{0} \sin \theta \cos \phi, k_{y}=k_{0} \sin \theta \sin \phi, z=0\right) \sin \varphi\right) \hat{\boldsymbol{\theta}} \\
& +\left(\widetilde{E}_{y}^{\mathrm{sc}}\left(k_{x}=k_{0} \sin \theta \cos \phi, k_{y}=k_{0} \sin \theta \sin \phi, z=0\right) \cos \varphi\right. \\
& \left.\left.-\widetilde{E}_{x}^{\mathrm{sc}}\left(k_{x}=k_{0} \sin \theta \cos \phi, k_{y}=k_{0} \sin \theta \sin \phi, z=0\right) \sin \varphi\right) \cos \theta \hat{\boldsymbol{\varphi}}\right] .
\end{aligned}
$$

Once the MoM described in Sec. III A has been applied and the system of equations (38) has been solved to obtain the unknown coefficients $e_{j l}$, Eqs. (47) and (48) make it possible to obtain the electric field radiated by the finite periodic array of slots. Maximum radiation is obtained in the positive $z$ direction, which is the propagation direction of the wave impinging on the array. The higher the number of slots, the more directional the maximum. A measurement of this directionality is given by the bistatic radar cross section of the screen in the positive $z$ direction, which is defined as $[23,33]$

$$
\sigma=\lim _{r \rightarrow \infty} \frac{4 \pi r^{2}\left|\mathbf{E}^{\mathrm{sc}}(r, \theta=0, \phi)\right|^{2}}{\left|\mathbf{E}_{i}\right|^{2}} .
$$

According to antenna theory, the radar cross section of the finitely perforated screen $\sigma$ will be the effective receiving area of the screen $A_{\mathrm{ef}}$ times the gain $G_{a}$ of the perforated screen when it radiates as an antenna in the half-space $z>0$. This effective receiving area times the Poynting vector of the incident wave will give the power transmitted to the half-space $z>0$. This means $A_{\mathrm{ef}}$ represents the area used by the incident wave for power transmission across the finite array of slots. When an aperture in the conducting screen is uniformly illuminated (which is the case considered in this paper), it turns out that [33]

$$
A_{\mathrm{ef}}=\frac{\lambda_{0}^{2}}{4 \pi} G_{a} .
$$

Therefore, under the conditions used in this paper, in which a uniform plane wave impinges on the finite array of slots, we can obtain $A_{\text {ef }}$ in terms of the radar cross section $\sigma$ as shown below,

$$
A_{\mathrm{ef}}=\sqrt{\frac{\sigma \lambda_{0}^{2}}{4 \pi}}
$$




\section{Basis functions and computation of Galerkin's matrix coefficients}

The basis functions we propose for $\mathbf{E}_{t}^{\mathrm{sc}}(x, y, z=0)$ in the slot $\eta_{j}$ are

$$
\begin{gathered}
\mathbf{d}_{j l}(x, y)=g_{l x}^{e e}\left(x-x_{c j}, y-y_{c j}\right) \hat{\mathbf{x}} \\
\left(j=1, \ldots, M ; l=1, \ldots, N_{b x}^{e e}\right) \\
\mathbf{d}_{j, N_{b x}^{e e}+l}(x, y)=g_{l x}^{e o}\left(x-x_{c j}, y-y_{c j}\right) \hat{\mathbf{x}} \\
=q_{x}^{e}\left(x-x_{c j}\right) r_{l x}^{o}\left(y-y_{c j}\right) \hat{\mathbf{x}}=q_{x}^{e}\left(x-x_{c j}\right) \\
\times U_{2 l-1}\left(\frac{2}{l_{s}}\left(y-y_{c j}\right)\right) \sqrt{1-\left[\frac{2}{l_{s}}\left(y-y_{c j}\right)\right]^{2}} \hat{\mathbf{x}} \\
\left(j=1, \ldots, M ; l=1, \ldots, N_{b x}^{e o}\right), \\
\mathbf{d}_{j, N_{b x}^{e e}+N_{b x}^{e o}+l}(x, y)=g_{l x}^{o e}\left(x-x_{c j}, y-y_{c j}\right) \hat{\mathbf{x}} \\
=q_{x}^{o}\left(x-x_{c j}\right) r_{l x}^{e}\left(y-y_{c j}\right) \hat{\mathbf{x}}=\frac{T_{1}\left(\frac{2}{w_{s}}\left(x-x_{c j}\right)\right)}{\sqrt{1-\left[\frac{2}{w_{s}}\left(x-x_{c j}\right)\right]^{2}}} \\
\times r_{l x}^{e}\left(y-y_{c j}\right) \hat{\mathbf{x}} \\
\left(j=1, \ldots, M ; l=1, \ldots, N_{b x}^{o e}\right), \\
\mathbf{d}_{j, N_{b x}^{e e}+N_{b x}^{e o}+N_{b x}^{o e}+l}(x, y)=g_{l x}^{o o}\left(x-x_{c j}, y-y_{c j}\right) \hat{\mathbf{x}} \\
=q_{x}^{o}\left(x-x_{c j}\right) r_{l x}^{o}\left(y-y_{c j}\right) \hat{\mathbf{x}} \\
\left(j=1, \ldots, M ; l=1, \ldots, N_{b x}^{o o}\right) \\
\quad \mathbf{d}_{j, N_{b x}+l}(x, y)=g_{l y}^{o o}\left(x-x_{c j}, y-y_{c j}\right) \hat{\mathbf{y}} \\
\left(j=1, \ldots, M ; l=1, \ldots, N_{b y}^{o o}\right) \\
(5 \\
(j, y)
\end{gathered}
$$

where $N_{b x}=N_{b x}^{e e}+N_{b x}^{e o}+N_{b x}^{o e}+N_{b x}^{o o}$, and where $N_{b x}+$ $N_{b y}^{o o}=N_{b}$. Once again, the basis functions of (52)-(56) are weighted Chebyshev polynomials to deal with the logarithmic singularities of the kernels of the set of integral equations (36) in the neighborhood of the slot edges [29].

By comparison with the infinite periodic case, the basis functions of (52)-(55) for the $x$ component of $\mathbf{E}_{t}^{\mathrm{sc}}(x, y, z=0)$ in the truncated periodic case not only contain functions that are even/even with respect to $x-x_{c j} / y-y_{c j}$, but they also contain functions that are even-odd, odd-even, and odd-odd with respect to $x-x_{c j} / y-y_{c j}$. And this is because the planes $x=x_{c j}$ and the planes $y=y_{c j}$ passing through the center of the slots in the truncated case are no longer electric walls and magnetic walls, respectively, and therefore the $x$ component of $\mathbf{E}_{t}^{\mathrm{sc}}(x, y, z=0)$ does not have any specific symmetry with respect to $x-x_{c j}$ and $y-y_{c j}$. To some extent, the basis functions of (53)-(55) are required in the truncated case to correct the current distribution provided in the infinite case by the basis functions of (52), especially in the slots that are closest to the array edges of Fig. 2. According to Eq. (56), we have used the same basis functions for the $y$ component of $\mathbf{E}_{t}^{\mathrm{sc}}(x, y, z=0)$ both in the infinite and the truncated case, which means we are not providing any correction for the $y$ component in the truncated case with respect to the infinite case. The explanation for this is that the $y$ component of $\mathbf{E}_{t}^{\mathrm{sc}}(x, y, z=0)$ is substantially smaller than the $x$ component

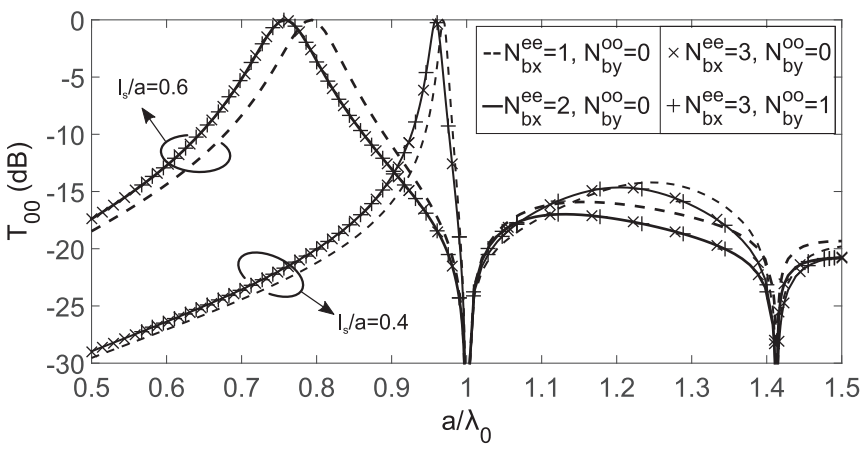

FIG. 3. Convergence pattern of the spatial domain MoM with respect to the number of basis functions (29) and (30) used in the approximation of the magnetic current density in the slots of Fig. 1. Results are presented for the power transmission coefficient $T_{00}$ of the transmitted fundamental wave. Parameters: $a=b$ and $w_{s} / a=0.05$.

when $w_{s} \ll a$ (this will be shown in Sec. IV), and therefore it does not make sense to provide a very accurate approximation of the $y$ component in the truncated case.

For the basis functions $\mathbf{d}_{j l}(x, y) \quad(j=1, \ldots, M ; l=$ $\left.1, \ldots, N_{b}\right)$ of $(52)-(56)$, the cross-correlations $h_{i j}^{k l}(x, y)$ and $h_{i j}^{k l, d d}(x, y)$ of (44) and (45) can all be obtained as linear combinations of the functions $F_{p r, q v}\left(x-\left(x_{c i}-x_{c j}\right), y-\right.$ $\left.\left(y_{c i}-y_{c j}\right)\right)$ [the functions $F_{p r, q v}(x, y)$ were defined in (31)]. This causes the integrands of (43) to show logarithmic singularities inside the integration domain for $x=x_{c i}-x_{c j}$ and $y=y_{c i}-y_{c j}$. Also, the function $G_{\text {sca }}(x, y)$ of (46) has a singularity at $x=y=0$, as happened with the function $G_{\mathrm{sca}}^{\mathrm{per}}(x, y)$ of $(18)$. When $i=j$, the point $(x=0, y=0)$ is inside the integration domain of $\Delta_{i j}^{k l}$ in (43), and the integrand of these integrals presents a singularity at $x=y=0$, which comes simultaneously from $G_{\mathrm{sca}}^{\mathrm{per}}(x, y)$, and from both functions $h_{i j}^{k l}(x, y)$ and $h_{i j}^{k l, d d}(x, y)$. This singularity has to be extracted in the way shown in (34) and (35), and the integrals containing the singularity have to be computed as described in Appendix. The remaining integrals containing the integrand minus the singularity at $(x=0, y=0)$ have to be determined by means of MRW quadrature rules since they still contain logarithmic singularities at $x=0$ when $y \neq 0$ and $y=0$ when $x \neq 0$. In the case in which $i \neq j$, the singular point $(x=0, y=0)$ of $G_{\mathrm{sca}}(x, y)$ is outside the integration domain of $\Delta_{i j}^{k l}$ in (43). In that particular case, the singularity at $(x=0, y=0)$ does not have to be extracted, and it suffices to use MRW quadrature rules in the computation of the integrals in order to handle the logarithmic singularities at $x=x_{c i}-x_{c j}$ and $y=y_{c i}-y_{c j}$.

\section{NUMERICAL RESULTS}

In Fig. 3, we present the spatial domain MoM results obtained for the transmission coefficient $T_{00}$ of two different infinite periodic arrays of slots. The data obtained for $T_{00}$ are plotted in decibels $\left[\left.T_{00}\right|_{\mathrm{dB}}=10 \log \left(T_{00}\right)\right]$. In the figure, we study the convergence of the MoM with respect to the number of basis functions of (29) and (30) used in (6). The results show that two basis functions of (29) suffice to achieve convergence in the approximation of the $x$ component of $\mathbf{E}_{t}^{\mathrm{sc}}(x, y, z=0)$. To explain this, we have to consider that the planes $y=m b+b / 2$ 
( $m=\ldots,-1,0,1, \ldots)$ of Fig. 1 are all magnetic walls under normal incidence conditions for the type of excitation we have assumed [10], and therefore the resonances that can be excited in the slots are those for which the length $l$ is roughly an odd number times half a wavelength (resonances for which $l$ is roughly an even number times half a wavelength are banned by symmetry). Since the results presented in Fig. 3 are restricted to slots for which $0.15 \lambda_{0} \leqslant l_{s} \leqslant 0.9 \lambda_{0}\left(\lambda_{0}=2 \pi / k_{0}\right.$ is the free-space wavelength), only the first resonance $\left(l_{s}\right.$ roughly equal to half a wavelength) will be excited, and the functional shape of the magnetic current in the slots along the $y$ direction will not be very different from that existing in the first resonance. This means that only two basis functions of the type shown in (29) will be required for convergence. Additional basis functions of (29) would be required if the results were obtained for larger values of the ratio $l_{s} / \lambda_{0}$ (this is not the case for the results presented in this paper, where $l_{s} / \lambda_{0}$ has always been chosen to be smaller than 1.2). The results of Fig. 3 also show that the introduction of one basis function of (30) for the $y$ component of $\mathbf{E}_{t}^{\mathrm{sc}}(x, y, z=0)$ has a negligible effect on the convergence pattern. In fact, the numerical simulations have shown that the magnitude of this $y$ component is roughly three orders smaller than the magnitude of the $x$ component. It has been checked that 25 evaluations of the integrands in (35) (which corresponds to using five MRW quadrature points both in the $x$ and $y$ integration variables) suffice to provide $\Gamma_{i j}$ with three significant figures, and that 100 evaluations of the integrand (10 MRW quadrature points both in the $x$ and $y$ variables) ensure an accuracy of five significant figures. This gives an idea of the numerical efficiency of the spatial domain MoM described in Sec. II.

In Fig. 4, our spatial domain MoM results are compared with results obtained using the commercial software $\operatorname{CST}^{\circledR}$ [34]. Excellent agreement is obtained between the two sets of results, which helps to validate our MoM code. When two basis functions per slot are used $-N_{b x}^{e e}=2$ and $N_{b y}^{o o}=0-\mathrm{CST}^{\circledR}$ turns out to be around 200 times slower than our spatial domain MoM code in the same computer. This large CPU time ratio shows the benefit of implementing specific home-made software for the efficient analysis of the particular structures studied in this paper.

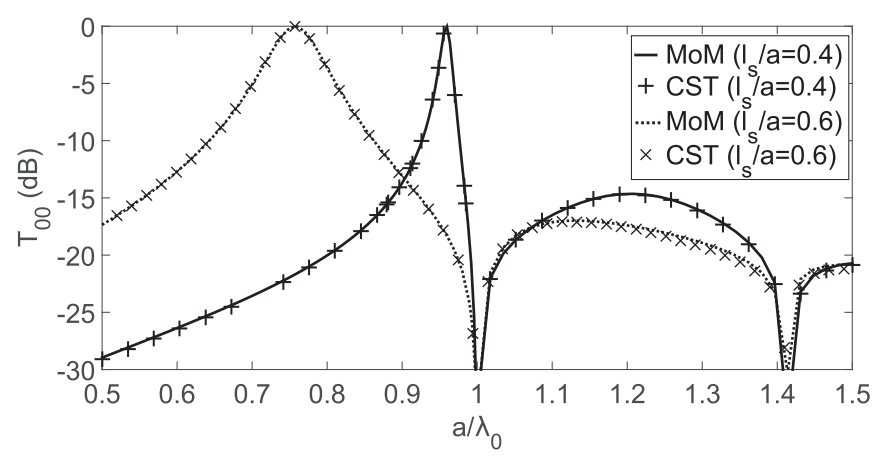

FIG. 4. Power transmission coefficient $T_{00}$ for the transmitted fundamental wave in infinite periodic arrays of slots. The results obtained with our MoM codes (solid line and dotted line) are compared with results provided by commercial software $\mathrm{CST}^{\circledR}(\times$ and +). Parameters: $a=b$ and $w_{s} / a=0.05$.

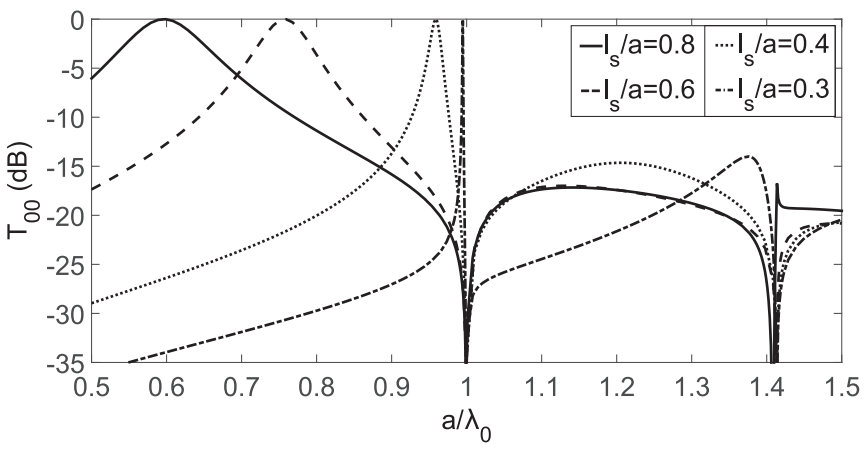

FIG. 5. Power transmission coefficient $T_{00}$ for the transmitted fundamental wave in infinite periodic arrays of slots. Results are presented for different slot lengths. Parameters: $a=b$ and $w_{s} / a=$ 0.05 .

Figure 5 shows the results obtained for the transmission coefficient $T_{00}$ of four different periodic arrays of slots. When the ratio between the length of the slot and the period, $l_{s} / a$, is larger than 0.5 , there is a first transmission peak when the slot is resonant and $l_{s} / \lambda_{0} \approx 0.5$, which is something to be expected. However, when $l_{s} / a<0.5$, there is always a first unexpected transmission peak for frequencies slightly smaller than the frequency of the first Wood's anomaly $\left(a / \lambda_{0}=1\right)$, which is called the extraordinary transmission peak [10]. In the case in which $l_{s} / a=0.4$, there is an extraordinary transmission peak at $a / \lambda_{0} \approx 0.96$, i.e., for a frequency smaller than that of the resonant transmission peak at $a / \lambda_{0} \approx 1.25$. In the case in which $l_{s} / a=0.3$, there is a first extraordinary transmission peak at $a / \lambda_{0} \approx 0.995$ very close to the first Wood's anomaly, and a second extraordinary transmission peak at $a / \lambda_{0} \approx 1.38$, which is also close to the second Wood's anomaly (occurring for $a / \lambda_{0}=\sqrt{2}$ ). These two peaks are located at frequencies smaller than the frequency of the resonant transmission peak at $a / \lambda_{0} \approx 1.67$. A detailed explanation of all these phenomena in terms of a convenient transmission line circuit model of the infinite periodic structure can be found in [10]. What seems to be clear about this is that the resonant transmission peaks are caused by resonances of the slots, and that the extraordinary transmission peaks are inherent to the periodicity of the structure of Fig. 1. Although the structure of Fig. 1 has an infinite number of cells, it is a fact that extraordinary transmission peaks have been experimentally detected in arrays of apertures with a finite number of cells [1,9,20,21]. In the rest of this section, we will investigate the appearance of extraordinary transmission peaks in truncated periodic arrays of slots (see Sec. III).

In Fig. 6, we show the results obtained with the spatial domain MoM of Sec. III for the effective receiving area of one truncated array of slots. The data for the effective receiving area are normalized to the surface occupied by the truncated periodic array of slots, $N^{2} a b$, and they are plotted in decibels $\left[A_{\mathrm{ef}} /\left.N^{2} a b\right|_{\mathrm{dB}}=20 \log \left(A_{\mathrm{ef}} / N^{2} a b\right)\right]$. As in the case of Fig. 3, in Fig. 6 we study the convergence of the MoM with respect to the number of basis functions of (52)-(56) used in (37). Despite the variety of basis functions introduced in Sec. III C, accurate results are obtained for the normalized effective receiving area when just using the first two basis functions of (52). As expected, numerical simulations have shown that 


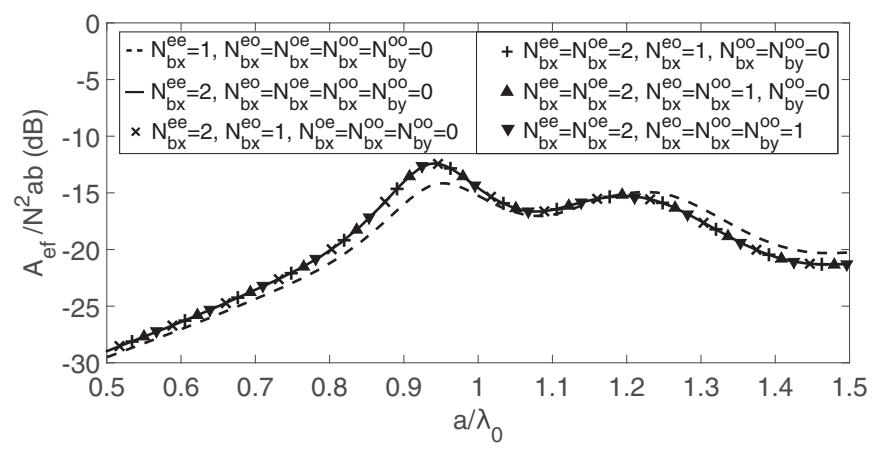

FIG. 6. Convergence pattern of the spatial domain MoM with respect to the number of basis functions (52)-(56) used in the approximation of the magnetic current density in a truncated periodic array of $5 \times 5=25$ slots. Results are presented for the normalized effective receiving area of the truncated periodic array. Parameters: $a=b, w_{s} / a=0.05$, and $l_{s} / a=0.4$.

the effect of the basis functions of (53)-(56) is negligible when approximating the magnetic current in the slots that are located around the center of the array of Fig. 2, just as happens in the case of the infinite periodic array. However, the basis functions of (53) and (54) provide a non-negligible correction to the basis functions of (52) when approximating the magnetic current in the slots that are located at the edges of the truncated array. Concerning the basis functions of (55) and (56), it has been found that their effect is always negligible in the approximation of the magnetic current of all the slots in the truncated array. So, even though the basis functions of (53) and (54) are required to obtain an accurate approximation of the magnetic current in the edge slots, they are not crucial for the accurate determination of the effective receiving area, probably because this parameter has to do with the performance of the whole truncated array, and the basis functions of (52) provide a reasonably accurate approximation of the magnetic current in the majority of the slots of the array.

To validate the spatial domain MoM of Sec. III, in Fig. 7 our MoM results are compared with $\operatorname{CST}^{\circledR}$ results [34], and reasonable agreement is found. The discrepancies observed for low values of $a / \lambda_{0}$ are attributed to the fact that a finite perfectly conducting plane was used in $\operatorname{CST}^{\circledR}$ computations,

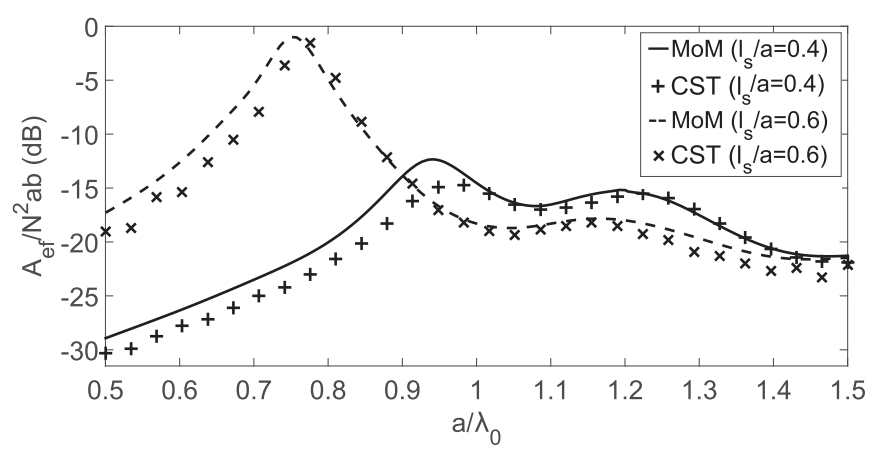

FIG. 7. Normalized effective receiving area of truncated periodic arrays of slots. The results obtained with our MoM code (solid line and dotted line) are compared with results provided by commercial software $\mathrm{CST}^{\circledR}\left(\times\right.$ and + ). Parameters: $a=b, w_{s} / a=0.05$, and $N=5$.

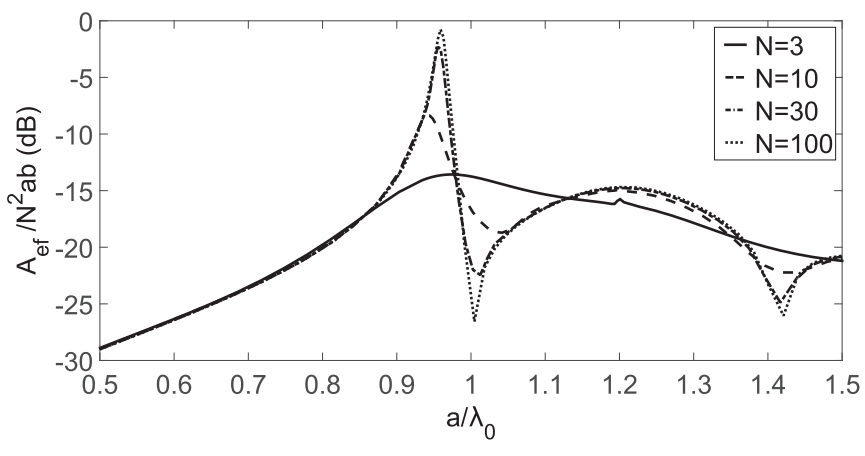

FIG. 8. Normalized effective receiving area of truncated periodic arrays of slots. Results are presented for increasing numbers of slots. Parameters: $a=b, w_{s} / a=0.05$, and $l_{s} / a=0.4$.

which unavoidably introduced some edge diffraction. In this case, $\operatorname{CST}^{\circledR}$ turns out to be around 100 times slower than our MoM code when five basis functions per slot were used $\left(N_{b x}^{e e}=N_{b x}^{o e}=2, N_{b x}^{e o}=1\right.$, and $\left.N_{b x}^{o o}=N_{b y}^{o o}=0\right)$. It should be pointed out that the computational burden of the truncated array of slots is much larger than that of the infinite array of slots. In fact, whereas the problem of the infinite array of slots can be reduced to analyzing one single cell due to the periodic boundary conditions, the analysis of a large truncated arrays of slots may involve the electromagnetic analysis of a surface with many squared wavelengths, and its computational burden increases nonlinearly as $N$ increases. In particular, numerical simulations have shown that whereas the analysis of an array of $20 \times 20$ slots in the range $0.5<a / \lambda_{0}<1.5$ typically requires a few minutes with our MoM code, the analysis of the same structure may require several hours with $\mathrm{CST}^{\circledR}$. So, the CPU time required to analyze large truncated arrays of slots $(N \geqslant 50)$ with $\operatorname{CST}^{\circledR}$ would be prohibitive. However, our MoM can handle these electrically large structures within reasonable CPU times.

Figure 8 shows the normalized receiving area of a truncated array of slots with normalized length $l_{s} / a=0.4$ as a function of the number $N$ of rows and columns involved in the array. Note that the extraordinary transmission peak and the two Wood's anomalies shown in Fig. 5 start to appear for $N=10$, and they are very well defined for $N=30$. In fact, if we compare Figs. 5 and 8, convergence from the finite case to the infinite case is nearly reached for $N=100$. To some extent, the normalized effective receiving area, $A_{\mathrm{ef}} / N^{2} a b$, is a measure of the percentage of the array area that the incident wave uses for power transmission. In the case in which $N=100$ and $a / \lambda_{0} \approx 0.96$, this percentage reaches $91.5 \%$ in Fig. 8 , which is very close to the result obtained in the infinite case in Fig. 5, where total transmission occurs.

In Figs. 9 and 10, we plot results for the ratio between the magnitude of the surface magnetic current (tangential electric field) at the center of one slot in a truncated periodic array and the magnitude of the surface magnetic current at the center of any of the slots of an infinite periodic array. This ratio is plotted as a function of the number of rows and columns in the truncated periodic array, $N$. The results are presented at the frequency for which $l_{s} / \lambda_{0}=0.5$, i.e., at the approximate frequency for which the slots are resonant. Due to the periodic boundary conditions, the magnitude of the surface magnetic 


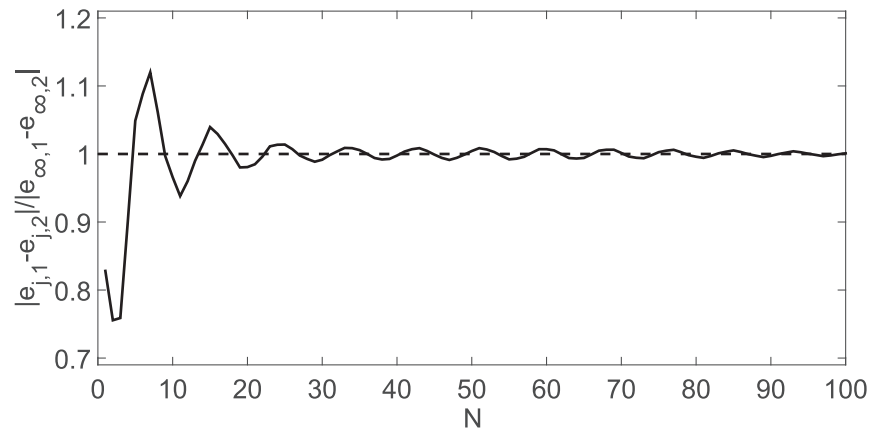

FIG. 9. Magnitude of the ratio between the surface magnetic current at the slot closest to the center of a periodic array of $N \times N$ slots (the center slot in case $N$ is odd, and one of the four slots closest to the array center in case $N$ is even) and the surface magnetic current at any of the slots of an infinite periodic array of slots. Parameters: $a=b, w_{s} / a=0.05, l_{s} / a=0.4$, and $a / \lambda_{0}=1.25$.

current is the same in all the slots of an infinite periodic array, but this is not the case in the truncated periodic array, as shown in Figs. 9 and 10. In the case of a slot next to the center of the periodic truncated array (Fig. 9), the surface magnetic current is essentially that existing in the slots of the infinite periodic array when $N \geqslant 20$. However, in the case of a slot placed at the corner of the truncated periodic array (Fig. 10), the surface magnetic current is always roughly $15 \%$ below that existing in the slots of the infinite periodic array, despite the value of $N$. This difference is due to edge effects in truncated periodic arrays. These edge effects have already been reported [35], and they have been found to introduce important differences between the scattering by infinite periodic structures and the scattering by truncated periodic structures, especially under oblique incidence conditions [36].

The edge effects at the boundaries of the truncated periodic array of slots are better visualized in Figs. 11 and 12. Figure 11 shows the normalized surface magnetic current at the slots of the 50th row of a truncated periodic array of $100 \times 100$ slots. Note that in the case of the 80 slots of the row closest to the center of the truncated array, the surface magnetic currents are basically those existing in the infinite array of the slots. This would help to explain why in Fig. 6 the basis

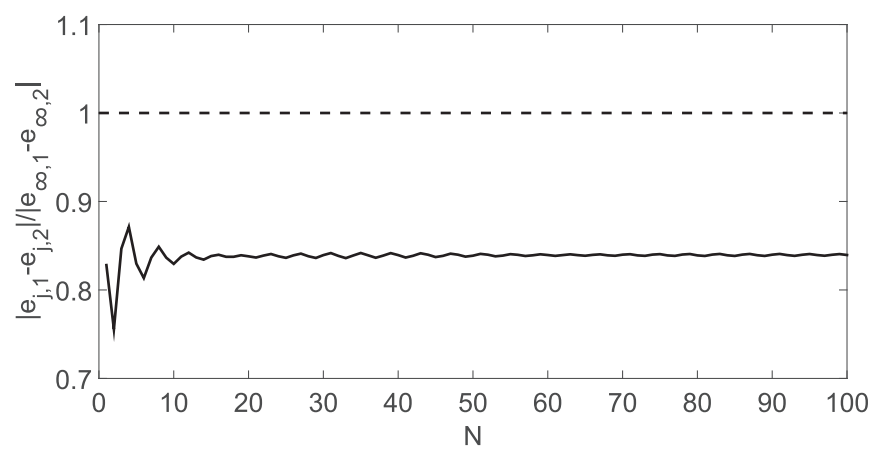

FIG. 10. Magnitude of the ratio between the surface magnetic current at the corner slot of a periodic array of $N \times N$ slots and the surface magnetic current at any of the slots of an infinite periodic array of slots. Parameters: $a=b, w_{s} / a=0.05, l_{s} / a=0.4$, and $a / \lambda_{0}=$ 1.25 .

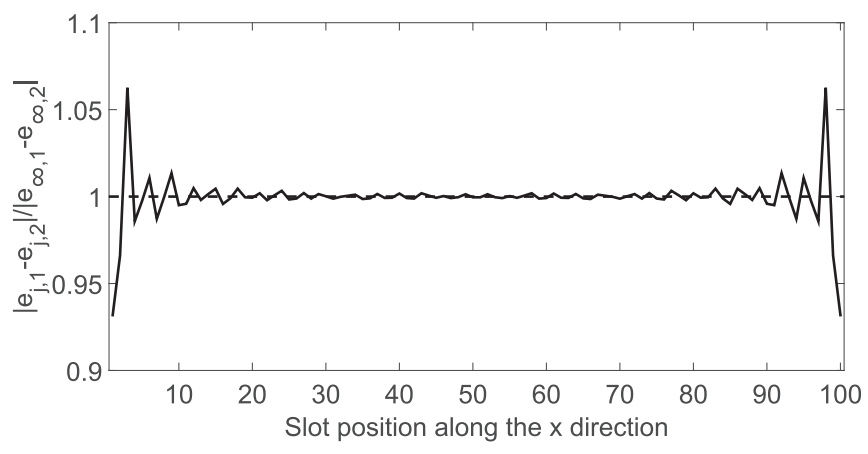

FIG. 11. Magnitude of the ratio between the magnetic current at the slots placed along the 50th row of a periodic array of $100 \times$ 100 slots and the magnetic current at any of the slots of an infinite periodic array of slots. Parameters: $a=b, w_{s} / a=0.05, l_{s} / a=0.4$, and $a / \lambda_{0}=1.25$.

functions used for the magnetic current in the infinite case suffice to provide accurate results for the normalized effective area in the truncated case. According to Fig. 11, only the slots that are closest to the edges show a magnetic current that is substantially different from that existing in the infinite case. Figure 12 provides an even better picture of the edge effects since it shows a complete $2 \mathrm{D}$ view of the surface magnetic current distribution in all the slots of the truncated array of $100 \times 100$ slots. Note that the magnetic current distribution is practically uniform around the center of the array, and it only experiences important deviations from that existing in the infinite case in the neighborhood of the edges and corners of the array.

The study carried out in Figs. 11 and 12 is repeated in Figs. 13 and 14 at a different frequency. Whereas the frequency used in Figs. 11 and 12 is the frequency for which the slots of the truncated array are roughly resonant $\left(l_{s} / \lambda_{0}=0.5\right.$ and $\left.a / \lambda_{0}=1.25\right)$, the frequency used in Figs. 13

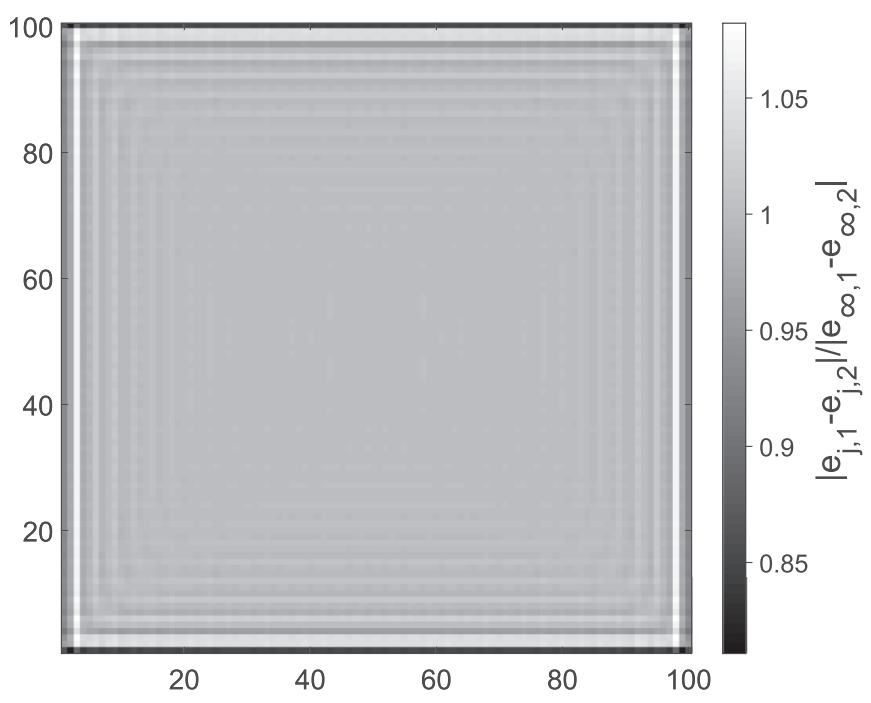

FIG. 12. Magnitude of the ratio between the magnetic current at the slots of a periodic array of $100 \times 100$ slots and the magnetic current at any of the slots of an infinite periodic array of slots. Parameters: $a=b, w_{s} / a=0.05, l_{s} / a=0.4$, and $a / \lambda_{0}=1.25$. 


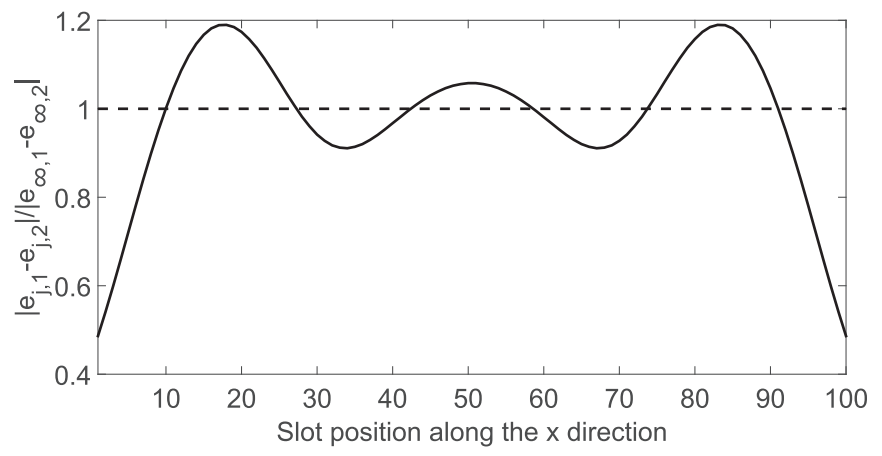

FIG. 13. Magnitude of the ratio between the magnetic current at the slots placed along the 50th row of a periodic array of $100 \times$ 100 slots and the magnetic current at any of the slots of an infinite periodic array of slots. Parameters: $a=b, w_{s} / a=0.05, l_{s} / a=0.4$, and $a / \lambda_{0}=0.959$.

and 14 is the frequency for which the truncated periodic structure experiences an extraordinary transmission peak (i.e., the frequency for which $a / \lambda_{0} \approx 0.96$, as shown in Fig. 8). Note that the picture shown in Figs. 13 and 14 is completely different from that shown in Figs. 11 and 12. In fact, the results of Figs. 13 and 14 show a standing-wave pattern along the $x$ axis of the array of Fig. 2, which suggests the existence of two magnetic current surface waves propagating along the array in opposite directions along the $x$ axis. This type of surface wave has been previously found in truncated periodic arrays at frequencies below the natural resonant frequencies of the elements of the array. In particular, Munk reports the existence of these waves [see Fig. 1.3(c) of [35]] in 2D periodic arrays of thin wires that are infinite in one direction and finite in the orthogonal direction. However, whereas Munk's current surface waves are excited when the direction of the incident waves and the plane of the array make an angle shorter than

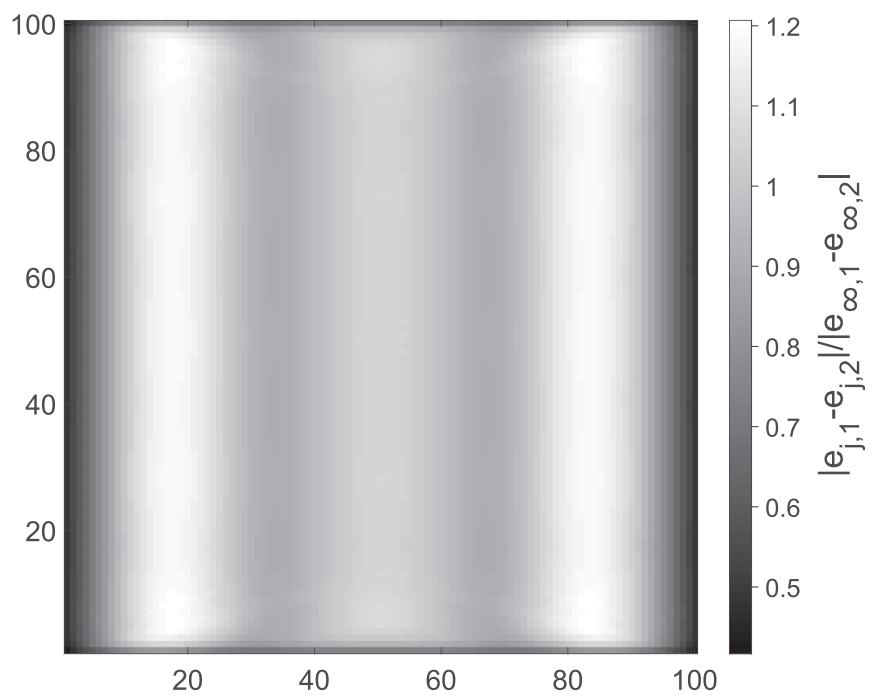

FIG. 14. Magnitude of the ratio between the magnetic current at the slots of a periodic array of $100 \times 100$ slots and the magnetic current at any of the slots of an infinite periodic array of slots. Parameters: $a=b, w_{s} / a=0.05, l_{s} / a=0.4$, and $a / \lambda_{0}=0.959$. $45^{\circ}$, the magnetic surface wave currents of Figs. 13 and 14 are excited for normal incidence.

\section{CONCLUSIONS}

In this paper, a very efficient spatial domain MoM is presented for the analysis of both infinite periodic arrays of slots and truncated periodic arrays of slots. The spatial domain MoM has been compared with the commercial software $\mathrm{CST}^{\circledR}$ both in the infinite case and in the truncated case, and good agreement has been found. The spatial domain MoM has been found to be around two orders of magnitude faster than $\operatorname{CST}^{\circledR}$ [34]. The spatial domain MoM has proven to be especially useful in the analysis of truncated periodic arrays of slots since it has made it possible to analyze arrays with $100 \times 100$ slots within reasonable CPU times. It has been verified that the results obtained for those large truncated arrays of slots converge to those obtained for infinite arrays. Also, well-known phenomena associated with periodicity such as extraordinary transmission and Wood's anomalies have been detected in small arrays containing only $10 \times 10$ slots. The magnetic current distribution in the slots of large truncated arrays has been found to be that existing in infinite arrays for the majority of the slots of the truncated arrays. However, important edge effects tend to appear for the slots close to the edges and corners of the truncated arrays. Finally, magnetic current surface waves have been observed in truncated arrays at the frequency of extraordinary transmission. Although these surface current waves were found previously in truncated arrays under oblique incidence conditions, in this case the surface current waves were detected under normal incidence conditions.

\section{ACKNOWLEDGMENTS}

This work has been supported by the Ministerio de Economía y Competitividad (Spain) with European Union FEDER funds in the frame of the project TEC2013-41913-P, and by the Consejería de Economía, Innovación, Ciencia y Empleo, Junta de Andalucía (Spain) in the frame of the project P12-TIC-1435.

\section{APPENDIX: EFFICIENT DETERMINATION OF SINGULAR INTEGRALS}

The constant coefficients $A_{p r, q v}^{i}(i=1, \ldots, 8)$ of (34) can be obtained in the case in which $p+r$ and $q+v$ are both even, as shown below,

$$
\begin{gathered}
A_{p r, q v}^{1}=\frac{C_{G}}{4 w_{s} l_{s}}\left[\ln \left(4 w_{s}\right)+u_{p r}\right]\left[\ln \left(4 l_{s}\right)+u_{q v}\right], \\
A_{p r, q v}^{2}=-\frac{C_{G}}{4 w_{s} l_{s}}\left[\ln \left(4 l_{s}\right)+u_{q v}\right], \\
A_{p r, q v}^{3}=-\frac{C_{G}}{4 w_{s} l_{s}}\left[\ln \left(4 w_{s}\right)+u_{p r}\right], \\
A_{p r, q v}^{4}=\frac{C_{G}}{4 w_{s} l_{s}}, \\
A_{p r, q v}^{5}=\frac{1}{16 \pi w_{s} l_{s}}\left[\ln \left(4 w_{s}\right)+u_{p r}\right]\left[\ln \left(4 l_{s}\right)+u_{q v}\right],
\end{gathered}
$$




$$
\begin{gathered}
A_{p r, q v}^{6}=-\frac{1}{16 \pi w_{s} l_{s}}\left[\ln \left(4 l_{s}\right)+u_{q v}\right], \\
A_{p r, q v}^{7}=-\frac{1}{16 \pi w_{s} l_{s}}\left[\ln \left(4 w_{s}\right)+u_{p r}\right], \\
A_{p r, q v}^{8}=\frac{1}{16 \pi w_{s} l_{s}},
\end{gathered}
$$

where $u_{p r}$ and $u_{q v}$ are numbers that can be obtained by means of the recurrent relations (42)-(47) of [16], and where the constant coefficient $C_{G}$ stands for the limit

$$
C_{G}=\lim _{x^{2}+y^{2} \rightarrow 0}\left[G_{\mathrm{sca}}^{\mathrm{per}}(x, y)-\frac{1}{4 \pi \sqrt{x^{2}+y^{2}}}\right] .
$$
is

This limit can be evaluated by means of (19), and the result

$$
\begin{aligned}
C_{G}= & \frac{1}{2 j a b} \sum_{m=-\infty}^{\infty} \sum_{n=-\infty}^{\infty} \frac{\operatorname{erfc}\left(j \sqrt{k_{0}^{2}-k_{x m}^{2}-k_{y n}^{2}} / 2 E\right)}{\sqrt{k_{0}^{2}-k_{x m}^{2}-k_{y n}^{2}}} \\
& +\frac{1}{8 \pi} \sum_{\substack{m=-\infty \\
|m|+|n| \neq 0}}^{\infty} \sum_{\substack{|c| \\
\text { (m }}}^{\infty} \frac{1}{\sqrt{m^{2} a^{2}+n^{2} b^{2}}} \\
& \times\left[e^{j k_{0} \sqrt{m^{2} a^{2}+n^{2} b^{2}}} \operatorname{erfc}\left(\sqrt{m^{2} a^{2}+n^{2} b^{2}} E+\frac{j k_{0}}{2 E}\right)\right. \\
& \left.+e^{-j k_{0} \sqrt{m^{2} a^{2}+n^{2} b^{2}}} \operatorname{erfc}\left(\sqrt{m^{2} a^{2}+n^{2} b^{2}} E-\frac{j k_{0}}{2 E}\right)\right] \\
& +\frac{k_{0}}{4 \pi j}\left[1-\operatorname{erfc}\left(\frac{j k_{0}}{2 E}\right)\right] .
\end{aligned}
$$

As happens with the double infinite summations of (19), the two double infinite summations of (A10) present Gaussian convergence, and it suffices to retain a small number of terms in each double summation to compute the series with great accuracy, which makes it possible to compute $C_{G}$ with great efficiency by means of (A10).

In the case in which $p+r$ and $q+v$ are both even, the second integral of (35) can be written as

$$
\int_{-w_{s}}^{+w_{s}} \int_{-l_{s}}^{+l_{s}} w_{p r, q v}^{\text {sing }}(x, y) d x d y=\sum_{i=1}^{8} A_{p r, q v}^{i} \Pi_{i},
$$

where

$$
\begin{gathered}
\Pi_{1}=4 w_{s} l_{s}, \\
\Pi_{2}=4 w_{s} l_{s}\left[\ln \left(w_{s}\right)-1\right], \\
\Pi_{3}=4 w_{s} l_{s}\left[\ln \left(l_{s}\right)-1\right], \\
\Pi_{4}=4 w_{s} l_{s}\left[\ln \left(w_{s}\right)-1\right]\left[\ln \left(l_{s}\right)-1\right], \\
\left.\int_{0}^{w_{s}} \int_{0}^{l_{s}} \frac{d x d y}{\sqrt{x^{2}+y^{2}}}\right]-4 w_{s} \ln \left[\frac{\sqrt{w_{s}^{2}+l_{s}^{2}}+l_{s}}{w_{s}}\right]
\end{gathered}
$$

(A16)

$$
\begin{gathered}
\Pi_{6}=4 \int_{0}^{w_{s}} \int_{0}^{l_{s}} \frac{\ln |x|}{\sqrt{x^{2}+y^{2}}} d x d y, \\
\Pi_{7}=4 \int_{0}^{w_{s}} \int_{0}^{l_{s}} \frac{\ln |y|}{\sqrt{x^{2}+y^{2}}} d x d y, \\
\Pi_{8}=4 \int_{0}^{w_{s}} \int_{0}^{l_{s}} \frac{\ln |x| \ln |y|}{\sqrt{x^{2}+y^{2}}} d x d y .
\end{gathered}
$$

The double integrals of (A17)-(A19) cannot be obtained in closed form. However, if polar variables are introduced $(x=$ $\rho \cos \varphi$ and $y=\rho \sin \varphi$ ), the integration with respect to $\rho$ can be carried out in closed form, and after some manipulations, $\Pi_{6}, \Pi_{7}$, and $\Pi_{8}$ can be expressed as

$$
\begin{aligned}
\Pi_{6}= & 4 w_{s}\left[\ln \left(w_{s}\right)-1\right] \ln \left[\frac{\sqrt{w_{s}^{2}+l_{s}^{2}}+l_{s}}{w_{s}}\right]+4 l_{s} \\
& \times\left\{\left(\Lambda_{1}-\Lambda_{2}\right)-\left[\ln \left(l_{s}\right)-1\right] \ln \left[\frac{\sqrt{w_{s}^{2}+l_{s}^{2}}-w_{s}}{l_{s}}\right]\right\}, \\
\Pi_{7}= & -4 l_{s}\left[\ln \left(l_{s}\right)-1\right] \ln \left[\frac{\sqrt{w_{s}^{2}+l_{s}^{2}}-w_{s}}{l_{s}}\right]+4 w_{s} \\
& \times\left\{\left(\Lambda_{3}-\Lambda_{4}\right)+\left[\ln \left(w_{s}\right)-1\right] \ln \left[\frac{\sqrt{w_{s}^{2}+l_{s}^{2}}+l_{s}}{w_{s}}\right]\right\}, \\
\Pi_{8}= & 4 w_{s}\left\{\left\{\left[\ln \left(w_{s}\right)\right]^{2}-21\right)\right. \\
& \left.+\left[\ln \left(w_{s}\right)-1\right]\left(\Lambda_{3}-\Lambda_{4}\right)\right\}+4 l_{s}\left\{\left[\ln \left(l_{s}\right)-1\right]\left(\Lambda_{1}-\Lambda_{2}\right)\right. \\
& -\left\{\left[\ln \left(l_{s}\right)\right]^{2}-2 \ln \left(l_{s}\right)+2\right\} \ln \left[\frac{\sqrt{w_{s}^{2}+l_{s}^{2}}+l_{s}}{w_{s}}\right] \\
l_{s} &
\end{aligned}
$$

The coefficients $\Lambda_{i}(i=1, \ldots, 4)$ of (A20)-(A22) are onedimensional integrals that cannot be obtained in closed form, and they are given by

$$
\begin{aligned}
& \Lambda_{1}=\int_{\varphi_{0}}^{\pi / 2} \frac{\ln (\cos \varphi)}{\sin \varphi} d \varphi=\int_{0}^{\cos \varphi_{0}} \frac{\ln t}{\left(1-t^{2}\right)} d t, \\
& \Lambda_{2}=\int_{\varphi_{0}}^{\pi / 2} \frac{\ln (\sin \varphi)}{\sin \varphi} d \varphi=\int_{\sin \varphi_{0}}^{1} \frac{\ln t}{t \sqrt{1-t^{2}}} d t, \\
& \Lambda_{3}=\int_{0}^{\varphi_{0}} \frac{\ln (\sin \varphi)}{\cos \varphi} d \varphi=\int_{0}^{\sin \varphi_{0}} \frac{\ln t}{\left(1-t^{2}\right)} d t, \\
& \Lambda_{4}=\int_{0}^{\varphi_{0}} \frac{\ln (\cos \varphi)}{\cos \varphi} d \varphi=\int_{\cos \varphi_{0}}^{1} \frac{\ln t}{t \sqrt{1-t^{2}}} d t,
\end{aligned}
$$

where $\varphi_{0}=\tan ^{-1}\left(l_{s} / w_{s}\right)$. The integrands of (A23) and (A25) present a logarithmic singularity when $t=0$, but the integrands of (A24) and (A26) do not present any singularities in the integration interval. Numerical simulations have shown that all the integrals $\Lambda_{i}(i=1, \ldots, 4)$ can be numerically computed with great accuracy by means of a low-order MRW quadrature rule. 
[1] T. W. Ebbesen, H. J. Lezec, H. F. Ghaemi, T. Thio, and P. A. Wolff, Nature (London) 391, 667 (1998).

[2] J. B. Pendry, L. Martín-Moreno, and F. J. García-Vidal, Science 305, 847 (2004).

[3] A. P. Hibbins, B. R. Evans, and J. R. Sambles, Science 308, 670 (2005).

[4] F. J. García-de Abajo, Rev. Mod. Phys. 79, 1267 (2007).

[5] C. Genet and T. W. Ebbesen, Nature (London) 445, 39 (2007).

[6] F. J. García-Vidal, L. Martín-Moreno, T. W. Ebbesen, and L. Kuipers, Rev. Mod. Phys. 82, 729 (2010).

[7] R. Gordon, A. G. Brolo, D. Sinton, and K. L. Kavanagh, Laser Photon. Rev. 4, 311 (2010).

[8] M. Beruete, M. Sorolla, I. Campillo, J. S. Dolado, L. MartínMoreno, J. Bravo-Abad, and F. J. García-Vidal, IEEE Trans. Antennas Propag. 53, 1897 (2005).

[9] M. Beruete, I. Campillo, M. Navarro-Cía, F. Falcone, and M. Sorolla, IEEE Trans. Antennas Propag. 55, 1514 (2007).

[10] F. Medina, F. Mesa, and R. Marqués, IEEE Trans. Microwave Theor. Tech. 56, 3108 (2008).

[11] H. A. Bethe, Phys. Rev. 66, 163 (1944).

[12] C.-C. Chen, IEEE Trans. Microwave Theor. Tech. 19, 475 (1971).

[13] R. Mittra, C. H. Chan, and T. Cwik, Proc. IEEE 76, 1593 (1988).

[14] B. A. Munk, Frequency Selective Surfaces: Theory and Design (Wiley, New York, 2000), p. 410.

[15] A. G. Schuchinsky, D. E. Zelenchuk, A. M. Lerer, and R. Dickie, IEEE Trans. Antennas Propag. 54, 490 (2006).

[16] R. Florencio, R. R. Boix, and J. A. Encinar, IEEE Trans. Antennas Propag. 63, 2558 (2015).

[17] M. Abramowitz and I. Stegun, Handbook of Mathematical Functions (Dover, New York, 1970).

[18] S. Hashemi-Yeganeh, IEEE Trans. Microwave Theor. Tech. 43, 641 (1995)
[19] R. R. Boix, M. Freire, and F. Medina, IEEE Trans. Antennas Propag. 52, 1080 (2004).

[20] F. Miyamuru and M. Hangyo, Appl. Phys. Lett. 84, 2742 (2004).

[21] F. Przybilla, A. Degiron, C. Genet, T. W. Ebbesen, F. de LeónPérez, J. Bravo-Abad, F. J. García-Vidal, and L. Martín-Moreno, Opt. Exp. 16, 9571 (2008).

[22] A. I. Fernández-Domínguez, F. J. García-Vidal, and L. MartínMoreno, Phys. Rev. B 76, 235430 (2007).

[23] R. F. Harrington, Time-harmonic Electromagnetic Fields (McGraw-Hill, New York, 1961).

[24] R. F. Harrington, Field Computation by Moment Methods (Wiley-IEEE, New York, 1993), pp. 1-21.

[25] D. G. Dudley, Mathematical Foundations for Electromagnetic Theory (Wiley-IEEE, New York, 1994).

[26] P. P. Ewald, Ann. Phys. (Leipzig) 64, 253 (1921).

[27] K. E. Jordan, G. R. Richter, and P. Sheng, J. Comput. Phys. 63, 222 (1986).

[28] S. Oroskar, D. R. Jackson, and D. R. Wilton, J. Comput. Phys. 219, 899 (2006).

[29] A. M. Lerer and A. G. Schuschinsky, IEEE Trans. Microwave Theor. Tech. 41, 2002 (1993).

[30] I. S. Gradshteyn and I. M. Rhyzik, Table of Integrals, Series and Products (Academic, San Diego, 2000), p. 795.

[31] R. Florencio, R. R. Boix, and J. A. Encinar, IEEE Trans. Antennas Propag. 61, 5088 (2013).

[32] J. Ma, V. Rokhlin, and S. Wandzura, SIAM J. Numer. Anal. 33, 971 (1996).

[33] R. E. Collin, Antennas and Radiowave Propagation (McGraw Hill, New York, 1985).

[34] CST, CST Microwave Studio, http://www.cst.com.

[35] B. A. Munk, Finite Antenna Arrays and FSS (Wiley Interscience, Hoboken, NJ, 2003).

[36] J. M. Usoff and B. Munk, IEEE Trans. Antennas Propag. 42, 946 (1994). 